\author{
Universidade de São Paulo
}

Instituto de Física

\title{
Fotodetectores de radiação infravermelha baseados em pontos quânticos de submonocamada
}

\author{
AHMAD AL ZEIDAN
}

\section{Orientador: \\ Prof. Alain André Quivy \\ Banca examinadora: \\ Prof. Fernando Josepetti Fonseca - LME - Poli \\ Prof. Mauricio Pamplona Pires - PUC/RJ}

Dissertação de mestrado apresentada ao Instituto de Física para a obtenção do título de Mestre em Ciências 


\section{FICHA CATALOGRÁFICA}

\section{Preparada pelo Serviço de Biblioteca e Informação do Instituto de Física da Universidade de São Paulo}

\section{Al-Zeidan, Ahmad}

Fotodetectores de radiação infravermelha baseados em pontos quânticos de submonocamada. São Paulo, 2017.

Dissertação (Mestrado) - Universidade de São Paulo. Instituto de Física. Depto. de Física de Materiais e Mecânica.

Orientador: Prof. Dr. Alain André Quivy

Área de Concentração: Física

Unitermos: 1. Fotodetectores; 2. Pontos quânticos; 3. Submonocamada; 4. InAs; 5. Infravermelho; 6. Epitaxia por feixes moleculares.

USP/IF/SBI-095/2017 


\title{
University of São Paulo \\ Institute of Physics
}

\section{Infrared photodetectors based on submonolayer quantum dots.}

\author{
AHMAD AL ZEIDAN \\ Supervisor: Prof. Dr. Alain André Quivy \\ Master thesis submitted to the Institute \\ of Physics to obtain the title of Master of Science
}

São Paulo

2017 



\section{Dedication}

It is with my deepest gratitude and warmest affection that I dedicate this thesis to my sweet family, especially to my parents Ibrahim and Mariam, and to my deceased son Abdul Rahman Al Zeidan for their endless love, support and encouragement. 


\section{Acknowledgements}

First of all, special thanks to Almighty God for giving me the strength to finish my dissertation.

Foremost, I would like to express my sincere gratitude to my supervisor, Prof. Alain André Quivy, for the continuous support during my master thesis, for his patience, motivation and immense knowledge. His guidance was helpful in all the steps of this thesis.

Secondly, my gratitude extends to Prof. Felix G. G. Hernandez, who supported me for the EUF 2014/2 when I arrived in Brazil.

I would like to thank Prof. Euzi C. F. da Silva for training me on the Wolfarm Mathematica to calculate the dark current.

I also would like to express my sincere thanks to my wife, Ghadeer Albattarni, for supporting me spiritually throughout this master program and my life in general.

To my friend Saeed Ullah, I am very deeply indebted for his unwavering enthusiasm, optimism and encouragement at every stage of my master degree. I am also grateful for the friendships that I formed inside USP.

Last, but not least, I would like to thank my whole family in mosque Vila São José, especially Abu Araújo, Khaled Alhamady, Mustafa Anis, Samir Hasani, Fuad Arisheh, Muhamed Jamus, Muhamed Sharif, Husaam Ali, Adnan and Abdul Hanan for their moral support. And there are much more and I am equally grateful to all of them.

I would also like to thank all the members of the MBE group at LNMS, especially Marcel Santos Claro for his support and help to do the experiments.

My special thanks also go to Prof. Paulo Nussenzveig, people of CPG (Andrea, Cláudia, Éber and Renata) and the secretaries of DFMT (Rosana, and Tatiana) for their support in all the official documents and correspondences.

I greatfully acknowledge the financial support from CNPq. 


\section{Resumo:}

Nesse trabalho, foi investigado um novo tipo de fotodetector de radiação infravermelha baseado em pontos quânticos de submonocamada de InAs obtidos pela técnica de epitaxia por feixe molecular (MBE, Molecular Beam Epitaxy). Suas propriedades foram comparadas com as de fotodetectores de pontos quânticos de InAs convencionais obtidos pela mesma técnica de deposição, mas no modo de crescimento StranskiKrastanov. Medidas de corrente de escuro, de ruído, de responsividade e de absorção mostraram que, dependendo da estrutura das amostras, os dispositivos com pontos quânticos de submonocamada podem ter um excelente desempenho.

\section{Palavras-chave:}

Fotodetectores, Pontos quânticos, Submonocamada, InAs, infravermelho, Epitaxia por feixes moleculares.

\section{Abstract:}

In this work, we investigated a new type of infrared photodetector based on InAs sub-monolayer quantum dots grown by molecular beam epitaxy (MBE). Their properties were compared with those of photodetectors containing conventional InAs quantum dots obtained by the same deposition technique, but in the Stranski-Krastanov growth mode. Dark current, noise, responsivity and absorption measurements have shown that, depending on the structure of the samples, the devices with sub-monolayer quantum dots can perform very well.

\section{Keywords:}

Photodetectors, Quantum dots, Submonolayer, InAs, Infrared, Molecular beam epitaxy 


\section{Table of contents}

Resumo / Abstract. $\quad$ V

Table of contents $\quad$ VII

List of figures. IX

List of tables. XIII

Nomenclature. $\quad$ XV

Introduction. $\quad 1$

Chapter 1: Infrared radiation \& detectors

1-1 Infrared radiation. 3

1-2 Infrared photodetectors. 6

1-2-1 Thermal detectors. 6

1-2-2 Photon detectors. 6

1-3 Quantum-well infrared photodetectors. 8

1-4 Quantum-dot infrared photodetectors. 10

\section{Chapter 2: Experimental methods}

2-1-1 Molecular-beam epitaxy. 13

2-1-2 Stranski-Krastanov quantum dots. 15

2-1-3 Sub-monolayer quantum dots. 16

2-2 Processing. 17

2-2-1 Optical lithography. 17

2-2-2 Metallization and packaging. 21

Electro-optical characterization

2-3 Dark current. 22

2-3-1 Experimental setup. 23

2-3-2 BLIP temperature. 24

2-4 Noise measurements. 25

2-4-1 Experimental setup. 26

2-5 Photocurrent with black body and responsivity experiment. 28

2-6 Spectral response. $\quad 30$ 


\section{Chapter 3: Photodetectors based on submonolayer quantum dots and on usual Stranski-Krastanov quantum dots.}

3-1 Design, growth \& processing of the samples 31

3-2 Electro-optical characterization 33

3.2.1 Dark current 34

3.2.2 Noise current 37

3.2.3 Photocurrent with a black body 38

3.2.4 Spectral Response 39

3-2-5 Responsivity 40

3-2-5 Specific detectivity 41

Chapter 4: photodetector based on submonolayer quantum dots in a quantum well.

4-1 Growth \& processing

4-2 Electro-optical characterization of the SML-QDWELL photodetector

\subsubsection{Dark Current}

4.2.2 Noise current

4.2.3 Photocurrent with black body

4.2.4 Spectral Response $\quad 50$

4-2-5 Specific detectivity $\quad 52$

Conclusion. $\quad 55$

$\begin{array}{ll}\text { Bibliography. } & 58\end{array}$ 


\section{List of Figures}

1-1 Schematics of the electromagnetic spectrum showing the range of the infrared spectrum and its absorption by the atmosphere.

1-2 Model of a black body

1-3 Spectral radiance as a function of wavelength $(\mu \mathrm{m})$ for a black body at different temperatures, illustrating Planck's Law. The red dashed line shows the shift of the emission peaks (Wien's Law).

1-4 Thermal imaging (infrared) illustrating (a) the thermal insulation faults of a house; (b) the presence of cancer in a breast region; (c) the detection of the turbine of a military jet.

1-5 Fundamental optical excitation processes in semiconductors: (a) intrinsic absorption from the valence to the conduction band, (b) extrinsic absorption from a donor impurity level (n-type doping) to the conduction band, and

(c) free-carrier absorption inside the conduction band

1-6 QWIPs can be obtained by depositing sequentially two semiconductor materials having different bandgaps. The material with the smaller bandgap (green) is the well and the material with the larger bandgap is the barrier (yellow).

1-7 Different types of structures and their degree of confinement of the carriers (a): bulk material with no confinement; (b): Quantum Well with a 1D confinement; (c) Quantum Wire with a 2D confinement; (d) Quantum Dot with a 3D confinement.

1-8 Band diagram of 2 QWIP structures: (a) bound to continuum and (b) bound to miniband. The three major mechanisms responsible for the dark current are also shown in (a): groundstate sequential tunneling (1), thermally assisted field-effect 10 tunneling (2), and thermionic emission (3). The gray regions indicate extended states through which the current can flow.

1-9 (a) Schematics of the main layers of a QWIP and a QDIP, (b) Schematics of a QDIP (or QWIP), showing its main 
components and operation mechanisms.

1-10 Schematic diagram of a typical vertical quantum-dot photodetector structure.

2-1 (a) Schematics of the growth chamber of a MBE system. (b) The MBE of the "Laboratório de Novos Materiais Semicondutores".

2-2 Scheme of SML-QD formation: (a) deposition of InAs submonolayers $(<1 \mathrm{ML})$ on top of GaAs to nucleate small 1 ML-high InAs islands; (b) Coverage of the InAs islands with GaAs material; (c) vertical alignment of the islands in consecutive InAs layers, (d) Full InGaAs QDs (blue box) formed by SML deposition (4 repetitions).

2-3 Clean room (ISO 6) of the "Laboratório de Novos Materiais Semicondutores".

2-4 Figure 2-4 [27]: Main steps of the processing of a photodetector: I sample, II photoresist layer, III mesa mask and exposure, IV development, V etching, VI removal of photoresist, VII photoresist, VIII contact mask and exposure, IX development, X metallization, XI lift off, XII wire bonding.

2-5 a) commercial chip carrier; b) Chip carrier with a sample fully processed.

2-6 The main components of the dark current: a) thermally excited electrons above the barrier; b) field-effect tunneling; c) direct tunneling.

2-7 a) Chip carrier containing the devices; b) chip carrier installed in the sample holder of the cryostat; c) $\mathrm{Cu}$ spring plate; d) $\mathrm{Cu}$ spring plate covering the chip carrier; e) dark shield surrounding the sample; f) cryostat fully mounted with $\mathrm{Ge}$ windows transparent in the IR spectrum (for further optical measurements).

2-8 Experimental setup for I-V experiment (dark current).

2-9 Experimental setup for the noise-current experiment. 
2-10 Spectral density of the noise voltage of a device at two different temperatures.

2-11 The experimental set up for the photocurrent / responsivity measurements.

2-12 Typical frequency spectrum of the total current obtained by the dynamic signal analyzer, showing the $1 / \mathrm{f}$ noise, the harmonics of the network, the noise from the cryogenic system, the signal of the photocurrent, and the noise from the 28 device (white noise).

2-13 Experimental setup for absorption measurements using FTIR spectroscopy.

2-14 Width at half maximum (determined by $\lambda 1$ and $\lambda 2$ ) of a typical absorption spectrum of a photodetector.

3-1 a) Structure of sample \#3551 (containing conventional SKQDs; b) of sample \#3601 (containing SML-QDs).

3-2 a) Photoluminescence spectrum of a sample of SML-QDs at $77 \mathrm{~K}$. b) PL spectra of a sample of SML-QDs at $180 \mathrm{~K}$ and of a InGaAs/GaAs quantum well with a similar composition and thickness.

3-3 (a) Cu spring plate; (b) $\mathrm{Cu}$ spring plate installed on top of the chip carrier and pressing it against the cold finger.

3-4 Dark-current curves of a device with (full lines) and without (dashed lines) the $\mathrm{Cu}$ spring plate.

3-5 Dark current of sample \#3551 (a) and \#3601 (b).

3-6 Dark current of sample \#3551 and sample \#3601 at low temperature.

3-7 Dark current as a function of temperature at a bias of $0.6 \mathrm{~V}$ for sample \#3551 (a) and \#3601 (b).

3-8 Band structure of a QDIP showing the activation energy, which is the difference of energy between the Fermi level EF and the top of the AlGaAs barriers. E0 is the theoretical energy required to excite an electron from the ground state of the quantum dot to the top of the AlGaAs barriers, and EQD 
is the calculated ground-state energy of the QDs.

3-9 Noise-current measurements as a function of bias voltage and temperature (a) for sample \# 3551 and (b) for sample \#3601. 38

3-10 Photocurrent as a function of bias and temperature using a black body for (a) sample \#3551 and (b) sample \#3601.

3-11 Spectral response (normalized) of sample \#3551 at $10 \mathrm{~K}$ for several bias voltages.

3-12 Black-body responsivity of sample \#3551 as a function of the bias voltage at $10 \mathrm{~K}$.

3-13 Specific detectivity of sample \# 3551.

4-1 Schematics of the structure of sample \#3691.

4-2 Schematics of the electrical connections between the devices and the pads.

4-3 Dark current of two different mesas \#2 (full) and \#3 (dashed) of sample \#3691 at different temperatures.

4-4 Dark current of mesa 2 of sample \#3691 showing also the I-V curve of the device obtained at $10 \mathrm{~K}$ when illuminated by a $300 \mathrm{~K}$ background (dashed, no $\mathrm{Cu}$ shield around the sample).

4-5 Dark current as a function of temperature at a bias of $1 \mathrm{~V}$.

4-6 Noise of the current inside the detector as a function of bias voltage and temperature for sample \#3691. The intensity of the noise was normalized by the bandwidth of the measurements for the further calculation of the specific detectivity.

4-7 Noise-current as a function of temperature at bias $=1 \mathrm{~V}$

4-8 Photocurrent measurements as a function of bias and temperature using a black body.

4-9 Spectral response (normalized) as a function of bias voltage at $10 \mathrm{~K}$ for positive and negative bias voltages. 
4-10 Black-body responsivity as a function of bias for different 52 temperatures.

4-11 Specific detectivity as a function of bias for different 52 temperatures. 


\section{List of Tables}

Table 1 Subdivision of the infrared spectrum. 3

Table 2 Comparison of various types of IR detectors. 10

Table 3 Comparison of the 3 growth methods. 15

Table 4 Comparison of both types of QDs. 54 


\section{List of abbreviations:}

IR

QDIPs

QWIPs

RTA

MBE

NIR

SWIR

MWIR

LWIR

VLWIR

FIR

LPE

CVD

RHEED

FM

VW

SK

SML

UV

SML-QDs

SK-QDs

BLIP

GR

FFT

FTIR

QWs

LNMS

sample \#3551

sample \#3601

sample \#3691
Infrared radiation.

Quantum-dot Infrared Photodetectors.

Quantum-well Infrared Photodetectors.

Rapid thermal annealing.

Molecular-beam epitaxy.

Near infrared.

Short-wavelength infrared.

Mid-wavelength infrared.

Long-wavelength infrared.

Very-long-wavelength infrared.

Far infrared.

Liquid-phase epitaxy.

Chemical vapor deposition.

Reflection high-energy electron diffraction.

Frank-Van der Merwe.

Volmer-Weber.

Stranski-Krastanov.

Sub-monolayer.

Ultra-violet.

Sub-monolayer quantum dots.

Stranski-Krastavov quantum dots.

Background limited infrared photodetector.

Generation-recombination.

Fast Fourier transform.

Fourier-transform infrared.

Quantum wells.

Laboratório de Novos Materiais Semicondutores.

Infrared photodetector based on usual Stranski-Krastanov quantum dots.

Infrared photodetectors based on submonolayer quantum dots.

Infrared photodetectors based on submonolayer quantum dots inserted inside a GaAs/AlGaAs quantum well. 


\section{List of symbols}

$\begin{array}{cl}\varepsilon & \text { Emissivity } \\ \mathrm{L}(\lambda, T) & \text { Spectral radiance } \\ h & \text { Planck's constant } \\ k & \text { Boltzmann's constant } \\ c & \text { Speed of light } \\ T & \text { Absolute temperature } \\ n_{t h} & \text { Density of thermal carriers } \\ \Phi_{B} & \text { Total background photon flux density } \\ t & \text { Detector's thickness. } \\ \tau & \text { Carrier lifetime } \\ \Delta f & \text { Bandwidth } \\ F & \text { Resistance of the detector (sample) } \\ i_{t h} & \text { Amplitude of the thermal noise as a current } \\ i_{G R} & \text { Amplitude of the GR noise as a current } \\ g_{n} & \text { photoconductive gain } \\ I_{d a r k} & \text { Dark current } \\ e & \text { Electron charge } \\ V_{\text {noise }} & \text { Noise voltage } \\ G & \text { Transimpedance gain } \\ I_{\text {noise }} & \text { Noise current } \\ P & \text { Input optical power } \\ I & \text { Photocurrent } \\ R & \text { Responsivity } \\ E_{\text {activ }} & \text { Activation energy } \\ D & \text { Detectivity } \\ D^{*} & \text { Specific detectivity } \\ A & \text { Optical area of the device } \\ & \end{array}$

\section{Physical constants:}

$\begin{array}{ll}e & \text { Electron charge } \\ k & \text { Boltzmann's constant } \\ c & \text { Speed of light } \\ h & \text { Planck's constant }\end{array}$

\footnotetext{
$1.60217662 \times 10^{-19} \mathrm{C}$$$
1.38064852 \times 10^{-23} \mathrm{~J} . \mathrm{K}^{-1}
$$$$
299792458 \mathrm{~m} . \mathrm{S}^{-1}
$$$$
6.626070040 \times 10^{-34} \mathrm{JS}
$$ 


\section{Introduction}

Infrared radiation (IR) is known since the early $19^{\text {th }}$ century, but it is only from the second half of the $20^{\text {th }}$ century that infrared detectors were developed (mainly for military applications) and that the development of this type of radiation has found some applications [1].

Most photodetector applications involve interband transitions in bulk materials, like $\mathrm{HgCdTe}$, or intraband transitions based on quantum-well structures [2]. The beginning of the interest in quantum-dot can be traced back to a suggestion by Arakawa and Sakaki in 1982 [3], that the performance of semiconductor lasers might be improved by reducing the dimensionality of the active regions of these devices. Ideal quantum dots should provide three-dimensional carrier confinement, resulting in discrete states for electrons and holes [4].

Quantum-dot Infrared Photodetectors (QDIPs) have recently emerged as a new technology for detecting infrared radiation. When compared to more conventional photodetectors based on quantum wells (QWIPs), their advantages originate from the three-dimensional confinement of carriers and include an intrinsic sensitivity to normal incidence of light, a longer lifetime of the photoexcited carriers and a lower dark current that should hopefully allow their operation near room temperature $[3,5]$

In the present work, several types of QDIPs were grown, processed and analyzed in order to check their performance. Two types of quantum dots were investigated: InAs quantum dots grown in the Stranski-Krastanov growth mode (SK-QDs), and InGaAs quantum dots obtained by the submonolayer technique (SML-QDs). All the samples were grown by molecular-beam epitaxy (MBE) at the "Laboratório de Novos Materiais Semicondutores" and then processed in a clean room using conventional photolithography techniques, e-beam metallization, rapid thermal annealing (RTA), and wire bonding. Finally, the optical and electrical properties of the devices were tested as a function of temperature and bias using darkcurrent curves, photocurrent measurements with a black body (to measure the responsivity), noise measurements with a signal analyzer (FFT), and absorption measurements using FTIR in order to calculate the specific 
detectivity. The growth of the 3 samples analyzed here was performed by a Ph.D student of our group (Marcelo Santos Claro), but I processed completely one of the samples and tested all of them again by myself to be sure that all the measurements would be performed in the same conditions. 


\section{Chapter 1: Infrared radiation \& detectors}

\section{1-1 Infrared radiation}

Before discussing infrared photodetectors, it is interesting to understand the nature and origin of the infrared radiation that is the part of the electromagnetic spectrum ranging from $0,74 \mu \mathrm{m}$ to $1000 \mu \mathrm{m}$. Its wavelength is longer than the one of visible light (figure 1-1) [6], meaning that infrared radiation is generally less scattered than visible light and offers better transmission through various media.

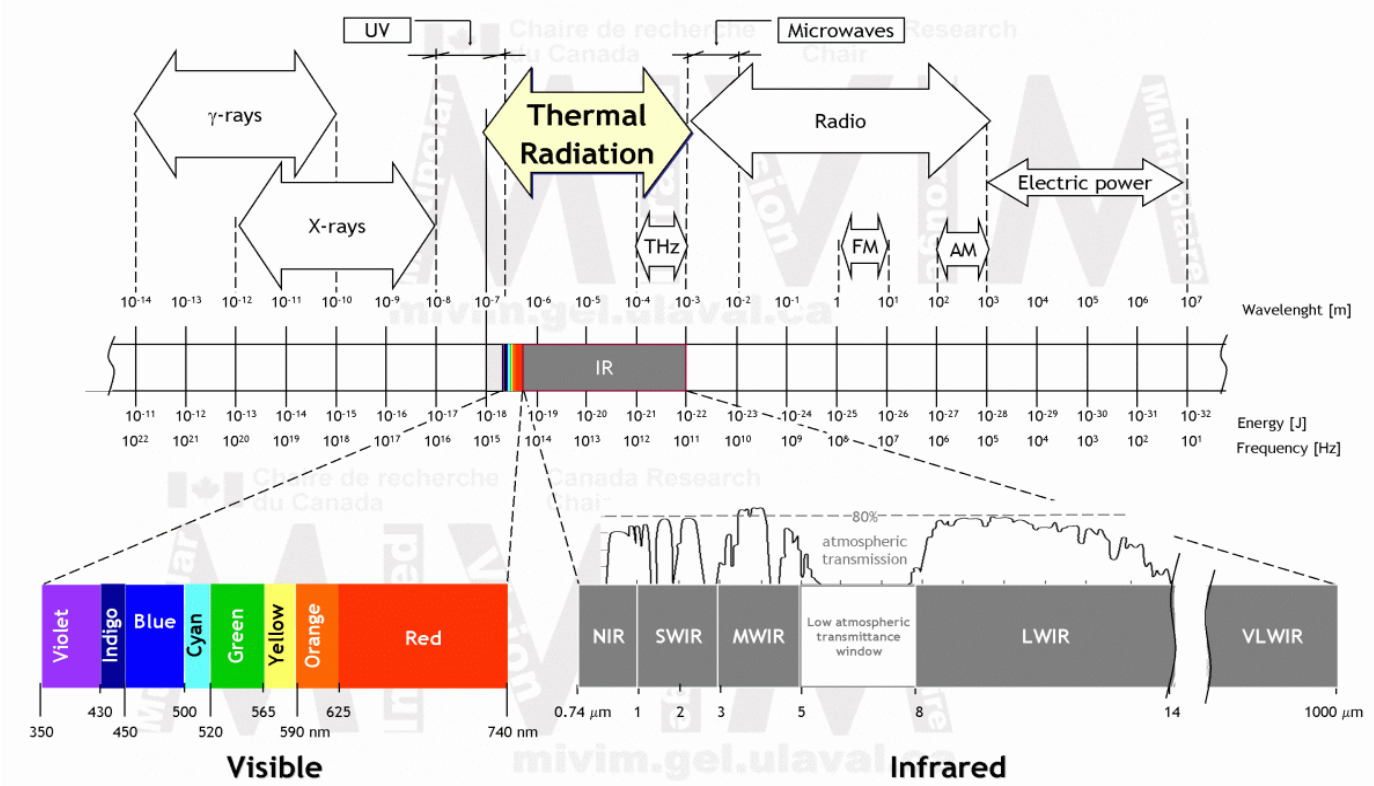

Figure 1-1: Schematics of the electromagnetic spectrum showing the range of the infrared spectrum and its absorption by the atmosphere.

The infrared spectrum is generally divided into six parts, depending on the wavelength (table 1). [7]

\begin{tabular}{|l|c|}
\hline Near Infrared (NIR) & $(0,74-1) \mu m$ \\
\hline Short-Wavelength Infrared (SWIR) & $(1-3) \mu m$ \\
\hline Mid-Wavelength Infrared (MWIR) & $(3-5) \mu m$ \\
\hline Long-Wavelength Infrared (LWIR) & $(8-14) \mu m$ \\
\hline Very-Long-Wavelength Infrared (VLWIR) & $(14-30) \mu m$ \\
\hline Far Infrared (FIR) & $(30-1000) \mu m$ \\
\hline Table 1: Subdivision of the infrared spectrum.
\end{tabular}


The research about infrared radiation is very important and interesting because any object with a temperature higher than zero Kelvin emits naturally an infrared radiation whose spectrum and intensity is a function of its temperature. It means that any object is a spontaneous source of IR radiation and can be directly observed with an IR camera, unlike the case of the visible spectrum where the human eye can only observe objects if some visible radiation is shined on them and scattered back to our eyes. In general, the infrared radiation emitted by an object depends on its temperature and on the properties of the surface.

This radiation is often approximated by the electromagnetic radiation emitted by an ideal body, called black body, which is an object that theoretically absorbs all the radiation that falls on it. A good approximation of a black body is a small hole leading to the inside of a hollow object that absorbs all the radiation that hits it and is in thermal equilibrium, as shown in figure 1-2 [8].

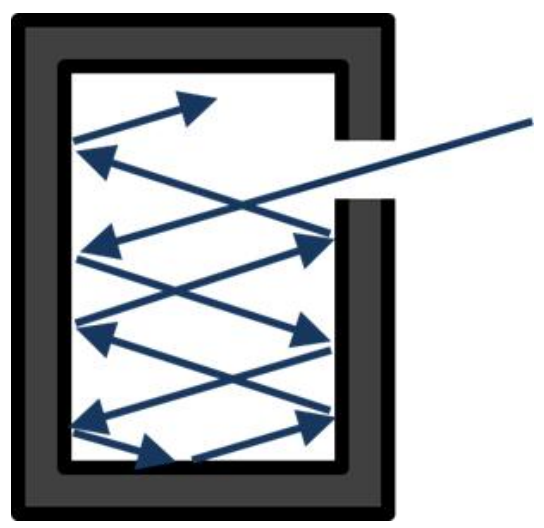

Figure 1-2: Model of a black body.

It turns out that a black body is also a perfect radiator and, therefore, the ratio of the radiant emittance $\mathrm{M}_{\text {object }}$ of a common object (radiant flux emitted by a surface per unit of surface and per unit of area) to that of a black body ( $\left.\mathrm{M}_{\text {black body }}\right)$ at the same temperature is the emissivity $\varepsilon$ that can be written as equation 1-1 [9]:

$$
\varepsilon=\frac{M_{\text {object }}}{M_{\text {black body }}} \ldots \ldots \ldots \ldots(1-1)
$$

Where, for an ideal black body, $\varepsilon=1$.

The spectral radiance $\mathrm{L}(\lambda, T)$ of a black body in thermal equilibrium at temperature $T$ is given by Planck's law $[9,10]$ : 


$$
L(\lambda, T)=\frac{2 \pi h c^{2}}{\lambda^{5}\left(e^{h c / \lambda k T}-1\right)} \ldots \ldots \ldots \ldots(1-2)
$$

Where $h$ is Planck's constant, $k$ is Boltzmann's constant and $c$ is the speed of light. It is shown in figure 1.3 for different temperatures [11]. It can be observed that the intensity of the curves increases with rising temperature, and that their maximum is shifted to lower wavelength, as determined by Wien's law $[9,10]$ :

$$
\lambda_{\max }=\frac{b}{T} \ldots \ldots \ldots \ldots(1-3)
$$

Where $b$ is a constant $\left(b=2.8977685 \times 10^{-3} \mathrm{~m} . K\right)$ and $\lambda_{\max }$ is the maximum of the spectral radiance at a specific absolute temperature $T$. Wien's Law shows that an object at $300 \mathrm{~K}$ emits an infrared radiation whose maximum is located at $9.65 \mu \mathrm{m}$ and is thus invisible to the human eye $[10,12]$. Above around $600{ }^{\circ} \mathrm{C}(873 \mathrm{~K})$, the intensity of the radiation increases considerably (with respect to $300 \mathrm{~K}$ ) and the emission spectrum starts to enter into the visible spectrum (figure 1-3) [12], leading to the natural incandescence of the bodies. In that specific case, objects start to emit visible (red) light spontaneously and they can be visible to the human eye (even in the dark) without shining any light at them.

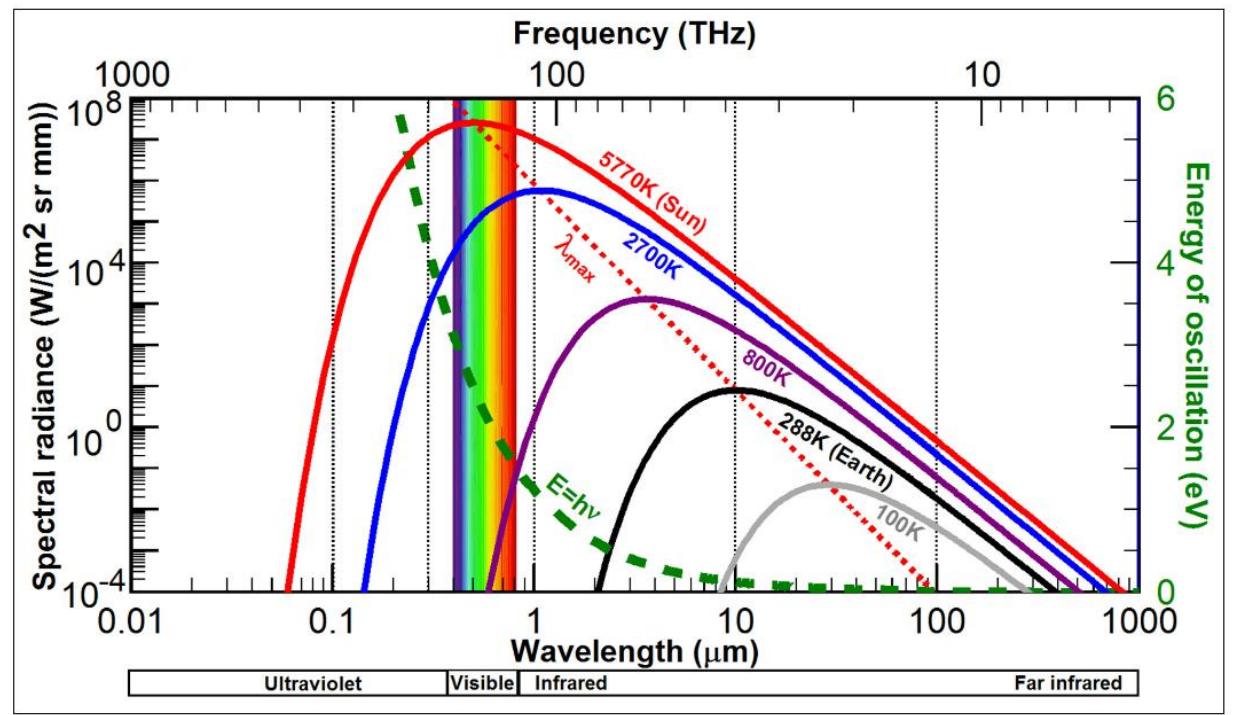

Figure 1-3: Spectral radiance as a function of wavelength $(\mu \mathrm{m})$ for a black body at different temperatures, illustrating Planck's law. The red dashed line shows the shift of the emission peaks (Wien's law). 


\section{1-2 Infrared detectors}

Today, with the manufacture of infrared detectors having a high sensitivity, high-quality infrared cameras are available and have applications in various strategic fields such as medicine, engineering, science, defense, agriculture, environment, and energy. We can see several examples of thermal images in figure 1-4 [13].

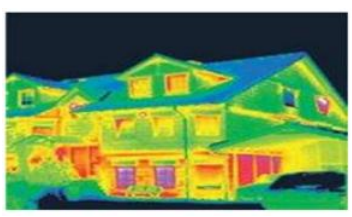

(a)

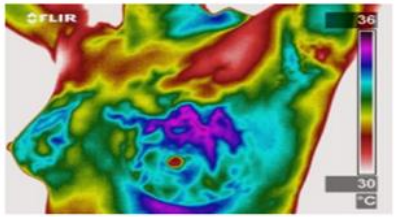

(b)

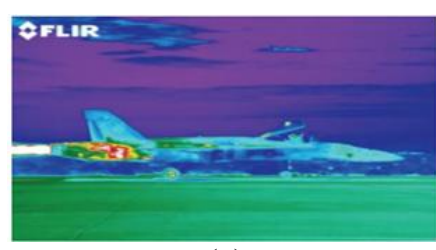

(c)

Figure 1-4: Thermal imaging (infrared) illustrating (a) the thermal insulation faults of a house; (b) the presence of cancer in a breast region; (c) the detection of the turbine of a military jet.

Infrared detectors can generally be classified into two different kinds, based on their intrinsic operation mechanisms: thermal detectors and photon detectors (also called photodetectors) [14].

\section{1-2-1 Thermal detectors}

In a thermal detector, the incident radiation is absorbed by the device and changes its temperature that, in turn, modifies the value of the parameter of the detector that is monitored. Depending on the property that is monitored (resistance, electric polarization), they have different names (bolometer, pyrometer). In this type of detectors, the output signal is independent of the incident wavelength because it depends only upon the radiant power [15]. Although they use to be slower than the other type of detector, they have the huge advantage to operate at room temperature, a feature that still makes them the device of choice for many applications in our daily routine.

\section{1-2-2 Photon detectors}

Photon detectors (that will hereafter be referred to as photodetectors) work in a different manner, as the infrared radiation is directly absorbed by electrons of the device that are promoted to higher energy levels and generate an electrical current (called photocurrent) that can be easily measured by an external circuit. Depending on their doping, these 
photodetectors are divided into 2 classes: intrinsic and extrinsic photodetectors [16].

\section{Intrinsic photodetectors}

Intrinsic photodetectors consist of semiconductors which have no intentional doping and thus are interband detectors [17, 18]. The incident photons must have an energy larger than the bandgap in order to generate an electron-hole pair whose components will transit to the contacts in different directions (figure 1-5-a) when a bias voltage is applied to the device. Their applications are usually limited by the energy gap of the materials that are available.

\section{Extrinsic photodetectors}

Extrinsic detectors consist of intentionally doped semiconductor materials in which the dopant elements generate an impurity level inside the band gap (figure 1-5-b) or in one of the bands (figure 1-5-c) [17, 18]. A photon hitting the surface of the detector can excite an electron from the impurity level to the conduction band that can contribute to the photocurrent (in the case of n-type doping). Since the energy transitions involved in this kind of photodetectors are generally small, these devices usually need to operate at low temperatures and therefore need cryogenic and vacuum components that make them bulky, heavy and more expensive.

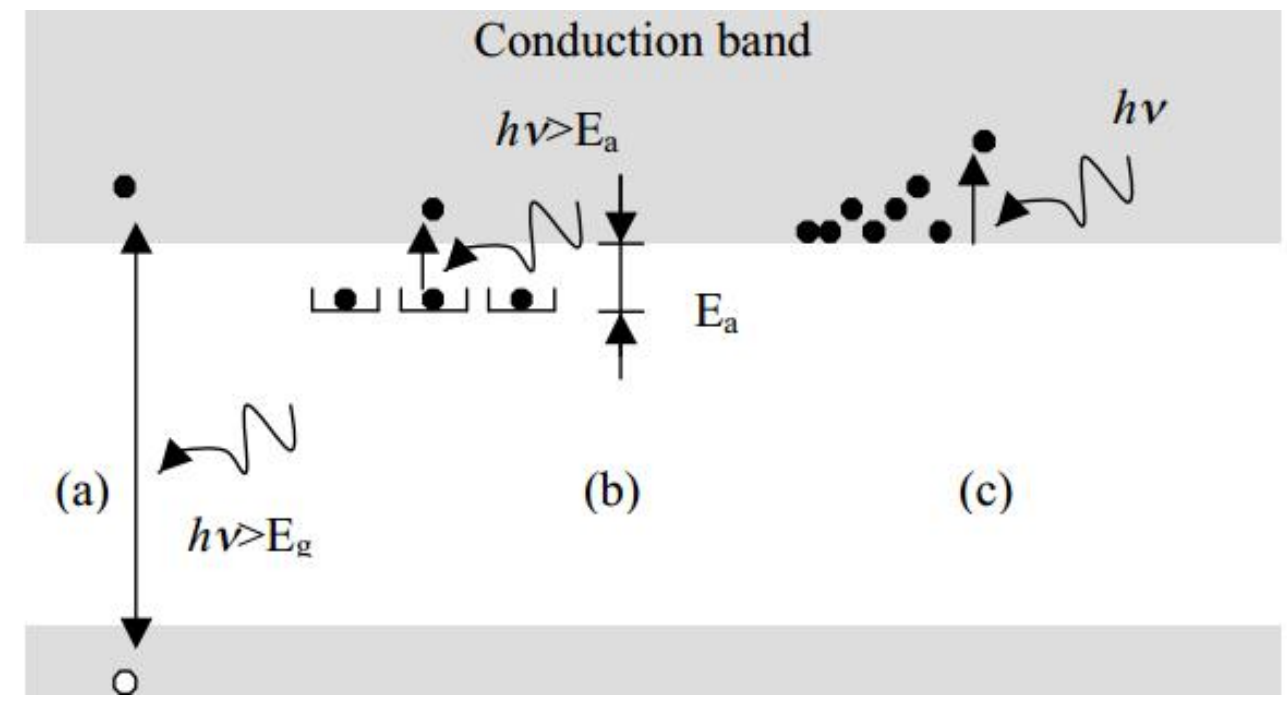

Figure 1-5: Fundamental optical excitation processes in semiconductors: (a) intrinsic absorption from the valence to the conduction band, (b) extrinsic absorption from a donor impurity level (n-type doping) to the conduction band, and (c) free-carrier absorption inside the conduction band [17]. 


\section{1-3 Quantum-Well Infrared Photodetectors}

Quantum-well Infrared Photodetectors (QWIPs) are based, as mentioned in their name, on quantum-well structures that basically consist of a thin layer of semiconductor material having a small bandgap which is surrounded by 2 layers of semiconductor materials having a larger bandgap. The material with the smaller bandgap is the "well" and the larger-bandgap material serves as the "barrier" of the well (figure 1-6-b) [19]. Since the well is generally a few $n m$ thick, the carriers are confined along one direction of space and their energy is quantized (figure 1-7-b). A QWIP is one of the simplest quantum mechanical device structures designed to detect midwavelength and long-wavelength infrared radiation $[20,21]$.

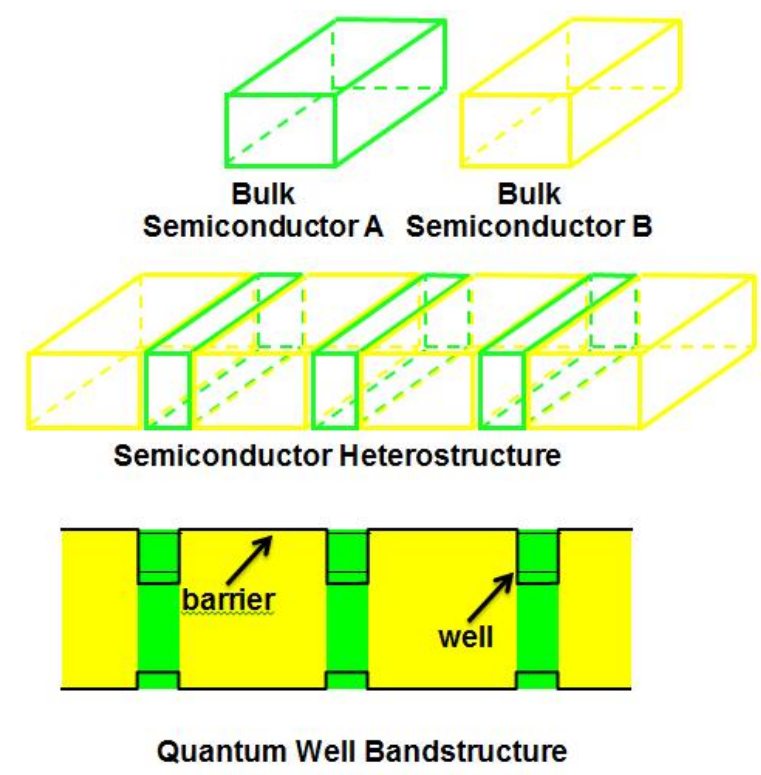

Figure 1-6: QWIPs can be obtained by depositing sequentially two semiconductor materials having different bandgaps. The material with the smaller bandgap (green) is the well and the material with the larger bandgap is the barrier (yellow). 


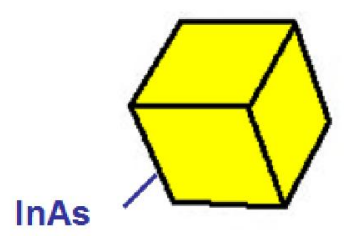

(a)

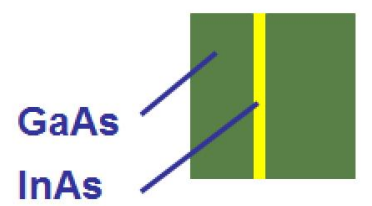

(c)

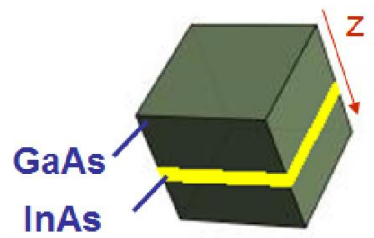

(b)

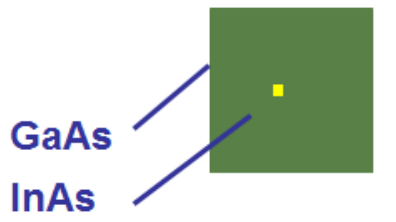

(d)

Figure 1-7: Different types of structures and their degree of confinement of the carriers (a): bulk material with no confinement; (b): Quantum Well with a 1D confinement; (c) Quantum Wire with a 2D confinement; (d) Quantum Dot with a 3D confinement.

To operate properly, the quantum wells of a QWIP need to be doped. For a n-type QWIP, electrons need to populate the lowest energy level of the QW and will be transferred to a higher energy level or to the continuum above the barrier whenever an IR photon with enough energy hits the surface of the device, generating a photocurrent. The absorption spectrum of the QWIP is determined by the optical transitions that are allowed in the quantum structure and depend directly on the nature of the materials involved in the device, their thickness and the bias voltage. By comparing the QWIPs with the other types of detectors (table 2, [15]) we can see that QWIPs have a good uniformity over a large area, multicolor capability, and easy wavelength control (by changing the width of the quantum well). However, the main disadvantages of QWIPs [19] are that they are not sensitive to normal incident radiation (due to intraband polarization selection rules), have high values of thermally generated dark current [15], and therefore require low (cryogenic) temperatures to operate properly, which makes them bulky, heavy and expensive as well. 


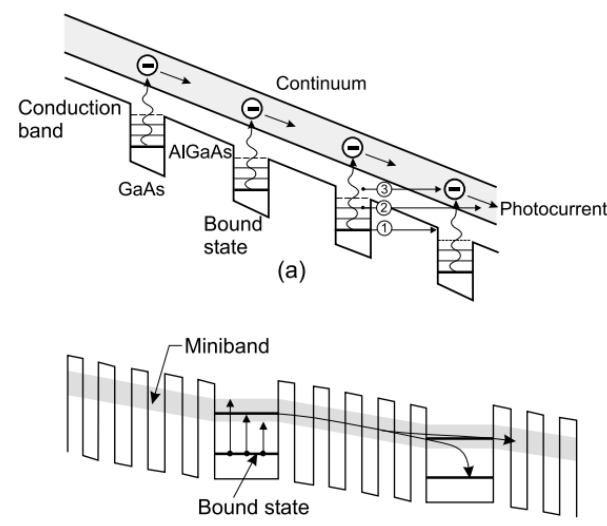

(b)

Figure 1-8: Band diagram of 2 QWIP structures: (a) bound to continuum and (b) bound to miniband. The three major mechanisms responsible for the dark current are also shown in (a): ground-state sequential tunneling (1), thermally assisted field-effect tunneling (2), and thermionic emission (3). The gray regions indicate extended states through which the current can flow [15].

\begin{tabular}{|c|c|c|}
\hline Detector type & Advantages & Disadvantages \\
\hline Thermal & $\begin{array}{l}\text { Light, rugged, reliable, and low cost } \\
\text { Room temperature operation }\end{array}$ & $\begin{array}{l}\text { Low detectivity at high frequency } \\
\text { Slow response (ms order) }\end{array}$ \\
\hline \multicolumn{3}{|l|}{ Photon } \\
\hline IV-VI & $\begin{array}{l}\text { Available low-gap materials } \\
\text { Well studied }\end{array}$ & $\begin{array}{l}\text { Poor mechanical } \\
\text { Large permittivity }\end{array}$ \\
\hline II-VI & $\begin{array}{l}\text { Easy band-gap tailoring } \\
\text { Well-developed theory and exp. } \\
\text { Multicolour detectors }\end{array}$ & $\begin{array}{l}\text { Non-uniformity over large area } \\
\text { High cost in growth and processing }\end{array}$ \\
\hline III-V & $\begin{array}{l}\text { Good material and dopants } \\
\text { Advanced technology } \\
\text { Possible monolithic integration }\end{array}$ & Heteroepitaxy with large lattice mismatch \\
\hline Extrinsic & $\begin{array}{l}\text { Very long wavelength operation } \\
\text { Relatively simple technology }\end{array}$ & Extremely low temperature operation \\
\hline Free carriers & $\begin{array}{l}\text { Low-cost, high yields } \\
\text { Large and close packed 2D arrays }\end{array}$ & $\begin{array}{l}\text { Low quantum efficiency } \\
\text { Low temperature operation }\end{array}$ \\
\hline \multicolumn{3}{|l|}{ Quantum wells } \\
\hline Type I & $\begin{array}{l}\text { Matured material growth } \\
\text { Good uniformity over large area } \\
\text { Multicolour detectors }\end{array}$ & $\begin{array}{l}\text { Low quantum efficiency } \\
\text { Complicated design and growth }\end{array}$ \\
\hline Type II & $\begin{array}{l}\text { Low Auger recombination rate } \\
\text { Easy wavelength control }\end{array}$ & $\begin{array}{l}\text { Complicated design and growth } \\
\text { Sensitive to the interfaces }\end{array}$ \\
\hline
\end{tabular}

Table 2: Comparison of various types of IR detectors.

\section{1-4 Quantum-dot Infrared Photodetectors (QDIPs)}

Since, in the nineties, it became possible to epitaxially grow quantum dots of good quality, some attempts were made to replace the quantum-well layers of a QWIP by quantum dots in order to solve the dark-current and normal-incidence problems encountered in QWIPs. Quantum-dot Infrared Photodetectors (QDIPs) are similar to QWIPs (figure 1-9-a) [3] and were 
expected to have improved performances because quantum dots (QDs) should have a fully discrete energy spectrum due to their small size along the three dimensions of space, as shown in figure 1-7.
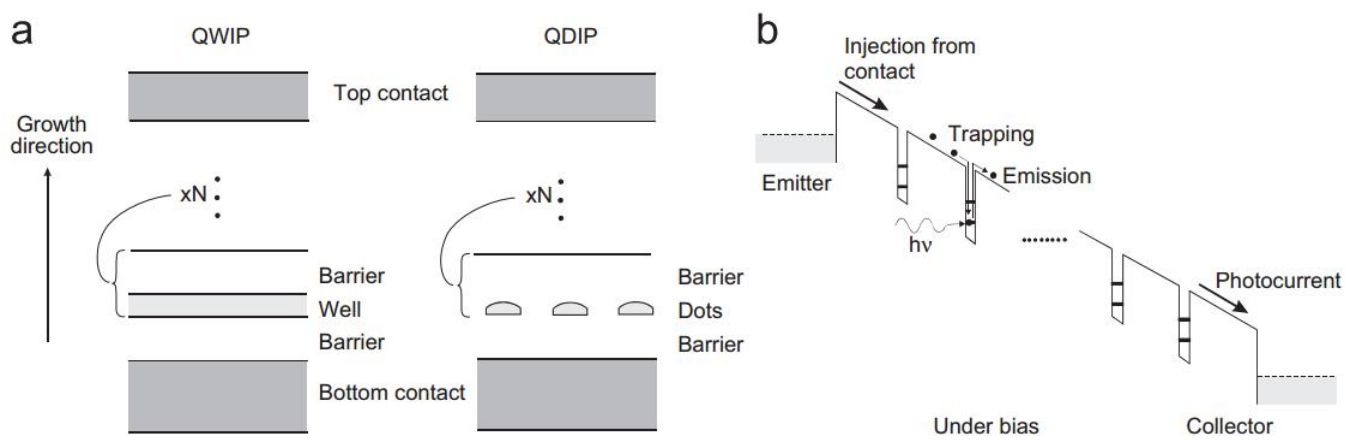

Figure 1-9: (a) Schematics of the main layers of a QWIP and a QDIP, (b) Schematics of a QDIP (or QWIP), showing its main components and operation mechanisms.

The advantages of QDIPs originate from the natural three-dimensional confinement of carriers (figure1-7-d) which leads to an intrinsic sensitivity to normal incidence of light, a longer lifetime of the photoexcited carriers (phonon bottleneck) and to a lower dark current that should hopefully allow their operation at higher temperature.

Two types of QDIPs structures can be found in the literature: vertical structures (where both electrical contacts are placed on top of each other) are very much investigated because they are more compact and adequate to build high-resolution focal-plane arrays that are used in IR cameras. Horizontal structures (both electrical contacts are side by side) have generally larger sizes and are preferred when the final performance is more important (i.e. in discrete detectors that will not be used for imaging) [3]. In this dissertation, only vertical devices were investigated, meaning that the carriers are transported vertically between the top and the bottom contacts (figure 1-10) [3].

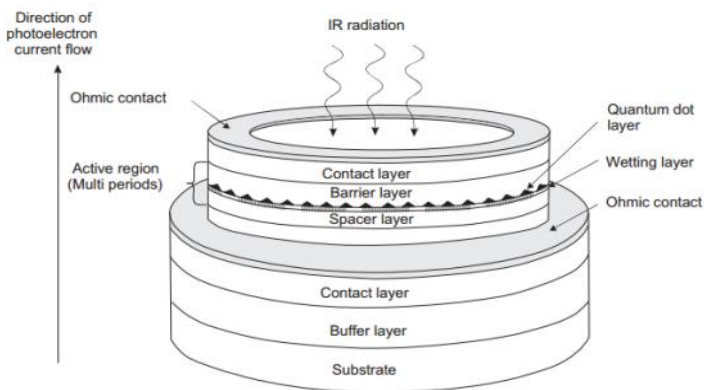

Figure 1-10: Schematic diagram of a typical vertical quantum-dot photodetector structure. 


\section{Chapter 2: $\quad$ Experimental methods}

In this chapter, I will discuss the main experimental techniques that were used to grow the samples, to process them into photodetectors, and to test the devices, as well as the major procedures and calculations performed to obtain reliable measurements.

\section{2-1-1 Molecular-beam epitaxy}

Many technologies can be used to produce thin films like the ones that are needed to fabricate the type of detectors that will be investigated here. Among them, we can cite thermal evaporation, sputtering, liquid-phase epitaxy (LPE), chemical vapor deposition (CVD), and molecular beam epitaxy (MBE) [22]. However, only the three last ones are able to provide single crystalline materials that are necessary to reach the desired performance. All the samples investigated in this work were grown in the MBE system of the "Laboratório de Novos Materiais Semicondutores". Molecular beam epitaxy was invented in the 1970s to produce epitaxial layers under ultra-high vacuum conditions in order to obtain heterostructures of compound semiconductors of high purity, high crystalline quality and having sharp interfaces [22].

The MBE system of the "Laboratório de Novos Materiais Semicondutores" is shown in figure 2-1-b and a typical growth chamber of a MBE system is shown in figure 2-1-a [23] and mainly consists of a vacuum chamber with a sample (wafer) holder that can be heated in order to allow the species adsorbed on the sample to diffuse and incorporate into the right site to provide the best crystalline quality. The sample holder can be rotated along several axes in order to bring the samples from another chamber in front of the cells. The cells contain all the high-purity material $(7 \mathrm{~N})$ that will be used for the epitaxy and need to be controlled individually in order to reach the right temperature able to provide the flux of material adequate to obtain the desired growth rate. Each cell has a shutter that can be opened or closed whenever needed to allow the growth of a specific material. The cells and the sample holder are surrounded by a panel containing liquid nitrogen that acts as an extra cryogenic pump to reduce further the pressure inside the chamber and to allow a better quality of the samples. Some insitu characterization techniques are also available. An infrared pyrometer allows the remote reading of the sample temperature, several Bayard- 
Alpert vacuum gauges are responsible for the measurement of the pressure inside the chamber and of the flux of materials, a mass spectrometer allows the analysis of the residual atmosphere, and a RHEED system (Reflection High-Energy Electron Diffraction), consisting of an electron gun and a fluorescent screen, allows the measurements of the growth rates and alloy composition in real time and the atomic surface reconstruction of the layers as a function of the growth conditions.

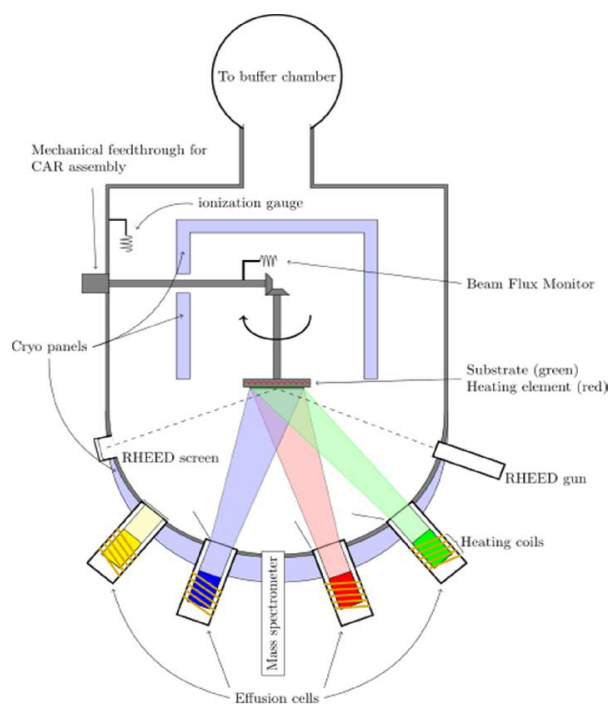

a

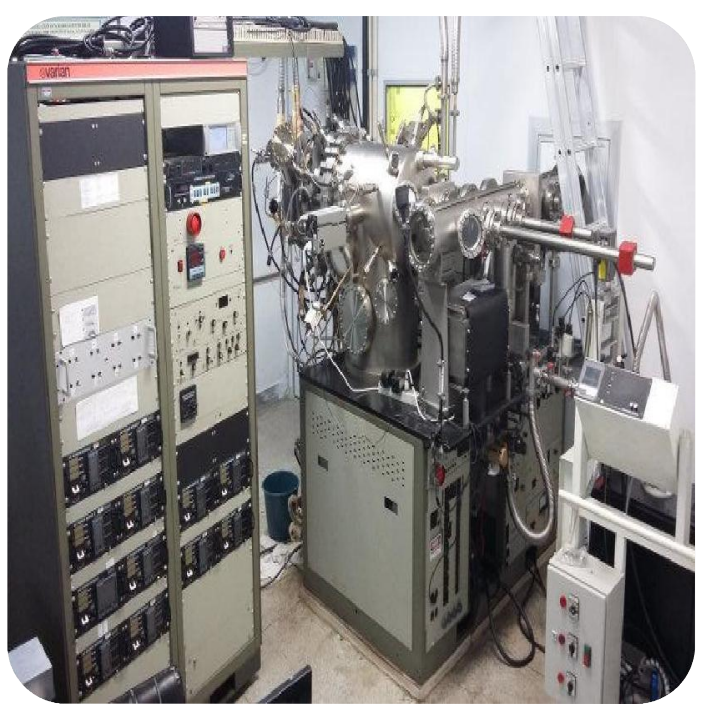

$\mathrm{b}$

Figure 2-1: (a) Schematics of the growth chamber of a MBE system. (b) The MBE of the "Laboratório de Novos Materiais Semicondutores".

There are basically three different growth modes to produce a thin film [23]: The Frank-van der Merwe (FM) growth mode is the one that is used for epitaxy of semiconductor materials, when the atomic layers are deposited one after each other, in a two-dimensional way, to produce an atomically flat surface. In the Volmer-Weber (VW) growth mode, which typically occurs when a metal is deposited on top of a semiconductor, large islands are formed at the surface and merge to cover the substrate with a continuous film. In the Stranski-Krastavov (SK) growth mode, which is used to grow self-assembled quantum dots, the deposition starts in the FM growth mode (that will lead to the formation of a wetting layer) and then switches to the VW growth mode due to an instability of the system (generally related to the accumulation of elastic energy due to the strain 
between the material and the substrate). Table 3 shows the comparison of the 3 growth modes.

\begin{tabular}{|c|c|c|}
\hline $\begin{array}{c}\text { Frank-van der Merwe } \\
\text { (FM) }\end{array}$ & Volmer-Weber (VW) & Stranski-Krastanov (SK) \\
\hline $\begin{array}{l}\text { Growth proceeds layer } \\
\text { by layer }\end{array}$ & $\begin{array}{l}\text { Growth causes three- } \\
\text { dimensional islands on } \\
\text { the substrate }\end{array}$ & $\begin{array}{l}\text { Starts in the FM mode } \\
\text { and then switches to the } \\
\text { VW mode. }\end{array}$ \\
\hline Epitaxial layer & & \\
\hline Substrate & Substrate & Substrate \\
\hline $\mathrm{a}$ & $\mathrm{b}$ & $\mathrm{c}$ \\
\hline
\end{tabular}

\section{2-1-2 Stranski-Krastanov Quantum Dots}

Self-assembled quantum dots are generally fabricated using the StranskiKrastanov growth mode during the deposition of a strained layer. The most well-known system consists of a thin InAs layer deposited on top of a GaAs substrate. Above a thickness of 1.7 MLs (monolayers), the thin InAs layer (that is under compressive strain) relaxes and spontaneously forms a high density of very small and homogeneous InAs islands that can confine the carriers along the 3 dimensions of space and thus behave as quantum dots. Since such nanostructures are self-assembled, they can only be controlled in a very limited way. They usually are lens shaped, have a density in the $10^{10} \mathrm{~cm}^{-2}$ range, and have a base and height of the order of $10-20 \mathrm{~nm}$ and $3-7 \mathrm{~nm}$ respectively. In this work, sample \#3551 was grown in the Stranski-Krastanov growth mode and will be used as a reference because this is the most common type of quantum dots that can be found in the literature.

\section{2-1-3 Sub-Monolayer Quantum Dots}

In order to compensate the lack of control of the SK-QDs, many research groups have been working on methods to improve the QDIP performance either by changing the composition of the QDs (InAs, InGaAs, InAlGaAs) or by changing the design of the structure using for example quantum dots 
in a well (DWELL), quantum dots in a double well (DDWELL) or quantum dot in a well with confinement barrier (CE DWELL).

In parallel, a new way to grow QDs was proposed in order to circumvent the control limitations of the SK-QDs which are intrinsic to the selfassembling process. Sub-Monolayer Quantum Dot (SML-QDs) are more difficult to grow but provide quantum dots with a higher density, with ondemand height, without wetting layer, and having improved threedimensional (3D) quantum confinement.

Such nanostructures were already used in vertical cavity surface-emitting lasers $[24,25]$ but only a few times in photodetectors [26]. The main idea to get $\operatorname{In}(\mathrm{Ga})$ As SML-QDs is to deposit a fraction of a monolayer of InAs material, generally between 30 and $50 \%$, in order to nucleate a high density of small two-dimensional (2D) islands on the GaAs substrate, and then to cover these islands with a specific number of GaAs monolayers. By repeating that sequence as many times as necessary (figure 2-2), a high density (up to $10^{12} \mathrm{~cm}^{-2}$ ) of InGaAs QDs having a wide range of height and composition can be obtained in a controllable way. Indeed, due to the elastic strain present in the InAs/GaAs system, the islands from the next InAs submonolayers will have a tendency to nucleate above the ones of the previous InAs submonolayers, thus forming stacks of InAs islands, separated by GaAs material, that will behave as individual InGaAs quantum dots. Since In segregates during GaAs capping, the InAs material of the 2D islands will be diluted in the stack and will form a single QD with an average InGaAs composition that will depend on the amount of InAs and GaAs material deposited in each cycle. The growth conditions of these SML-QDs are much more difficult to achieve because the small InAs islands will not be formed if the As flux is not considerably lowered in order to keep a $2 \times 4$ surface reconstruction during the whole deposition process of the SML-QDs (instead of the common $c 4 \times 4$ surface reconstruction which is obtained during the growth of the SK-QDs at $\left.500^{\circ} \mathrm{C}\right)$. 
(a)

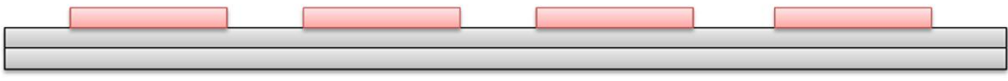

(b)

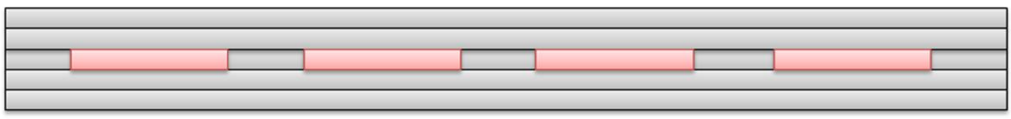

(c)

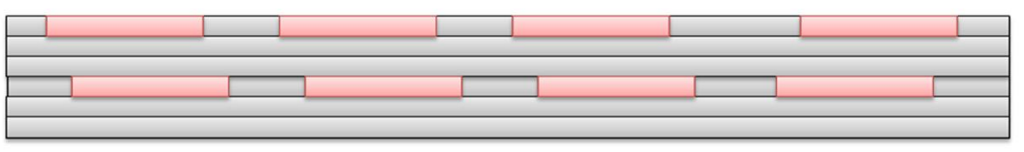

(d)

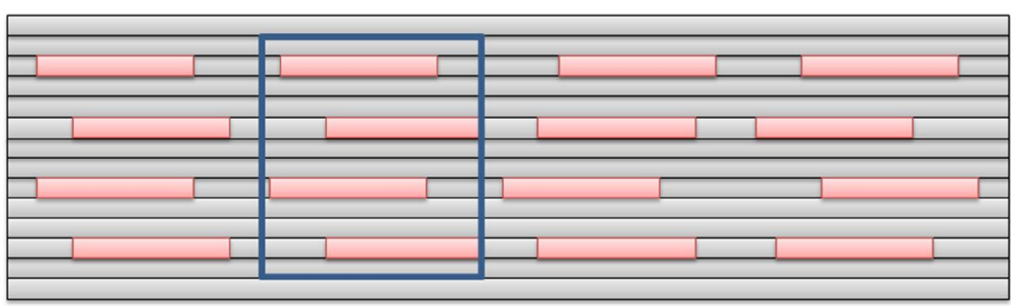

Figure 2-2: Scheme of SML-QD formation: (a) deposition of InAs submonolayers $(<1 \mathrm{ML})$ on top of GaAs to nucleate small 1 ML-high InAs islands; (b) Coverage of the InAs islands with GaAs material; (c) vertical alignment of the islands in consecutive InAs layers, (d) Full InGaAs QDs (blue box) formed by SML deposition (4 repetitions).

In this work, samples \#3691 \& \#3601 contain submonolayer quantum dots (SML-QDs) and will be compared to sample \#3551 which has SK-QDs.

\section{2-2 Processing}

All the samples analyzed here were processed in the same way. However, sample \#3551 and \#3601 were processed by previous students, while sample \#3691 was processed by myself during this work. Standard processing techniques were used to define small squared mesas using photolithography; electron-beam metallization and RTA (Rapid Thermal Annealing) allowed to make small Ohmic contacts, while wire bonding with thin Au wires was used to connect the devices to the chip carrier.

\section{2-2-1 Optical Lithography}

All the steps related to photolithography were accomplished in the new ISO 6 clean room of our laboratory (figure 2-3) [15]. To define the size of the mesas, the sample was placed in a spinner, covered by a few drops of photoresist (AZ5214) (figure 2-4-I and 2-4-II) and then rotated at $4000 \mathrm{rpm}$ during $30 \mathrm{~s}$ to produce a uniform layer of photoresist with a thickness around $1.4 \mu \mathrm{m}$. The sample was then heated (soft baking) at $90^{\circ} \mathrm{C}$ during $4 \mathrm{~min}$ on a hot plate to remove the solvent present in the 
photoresist. After the soft baking, the sample was placed in a mask aligner with a mask on top of it (figure 2-4-III). The mask consists of a glass plate covered with a thin metallic film containing the pattern that needs to be transferred to the sample. This metallic pattern will locally protect the photoresist from the ultra-violet (UV) radiation that is generated by the mask aligner during the exposure process. In the case of a positive photoresist, the regions of the photoresist layer that will be exposed to the UV light (during $9 \mathrm{~s}$ at $24 \mathrm{~mW} / \mathrm{cm}^{2}$ ) will react and will be removed during the development fase, while the regions that were protected by the metallic pattern of the mask will remain intact at the surface of the sample.

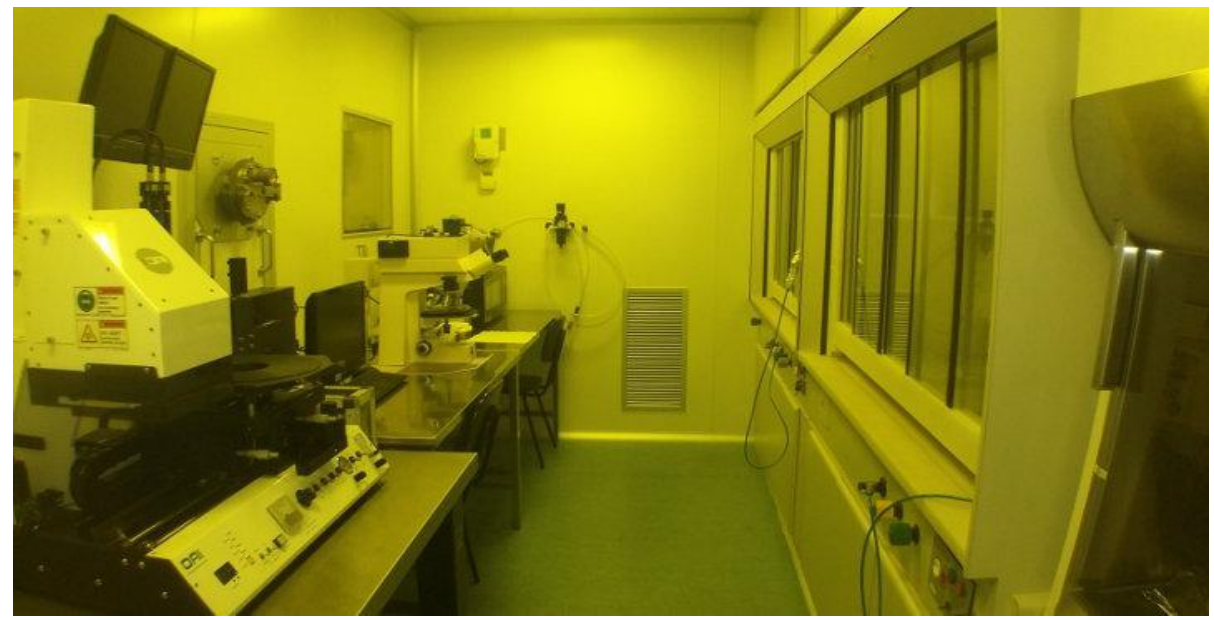

Figure2-3: Clean room (ISO 6) of the "Laboratório de Novos Materiais Semicondutores".

After the development (figure 2-4-IV), that consists in dipping the sample in a developer (AZ400: $\mathrm{H}_{2} \mathrm{O}$ [1:4]) during around $20 \mathrm{~s}$, the sample is rinsed in DI water, blown with dry nitrogen, and heated again (hard baking) at $120^{\circ} \mathrm{C}$ during $20 \mathrm{~min}$ in order to prepare the photoresist patterns that remained on the sample for the chemical etching (figure 2-4-V) that will remove the material of the sample everywhere the photoresist layer was absent. The etching solution was $\mathrm{H}_{2} \mathrm{O}_{2}: \mathrm{H}_{2} \mathrm{SO}_{4}: \mathrm{H}_{2} \mathrm{O}$ (1:8:40) and is known to etch GaAs at a rate close to $1 \mu \mathrm{m} / \mathrm{min}$. The actual etching rate was checked with a profiler in the sample itself, and the total etching was performed in 2 steps (after checking each step with the profiler) in order to reach precisely the middle of the bottom contact. After the removal of the photoresist pattern from the surface, using acetone and isopropanol, the sample contained a large number of small squared mesas having a lateral size of $400 \mu \mathrm{m} \times 400 \mu \mathrm{m}$ and a height that will depend on the etching 
time (figure 2-4-VI). The same steps mentioned in figure 2-4 II, III, IV were repeated once more to open a small window in the photoresist layer spread on top of every mesa using another mask (figure 2-4 VII, VIII, IX). This small window (figure 2-4-IX) shows the place where the small electrical contacts will be deposited in the next step (metallization). 


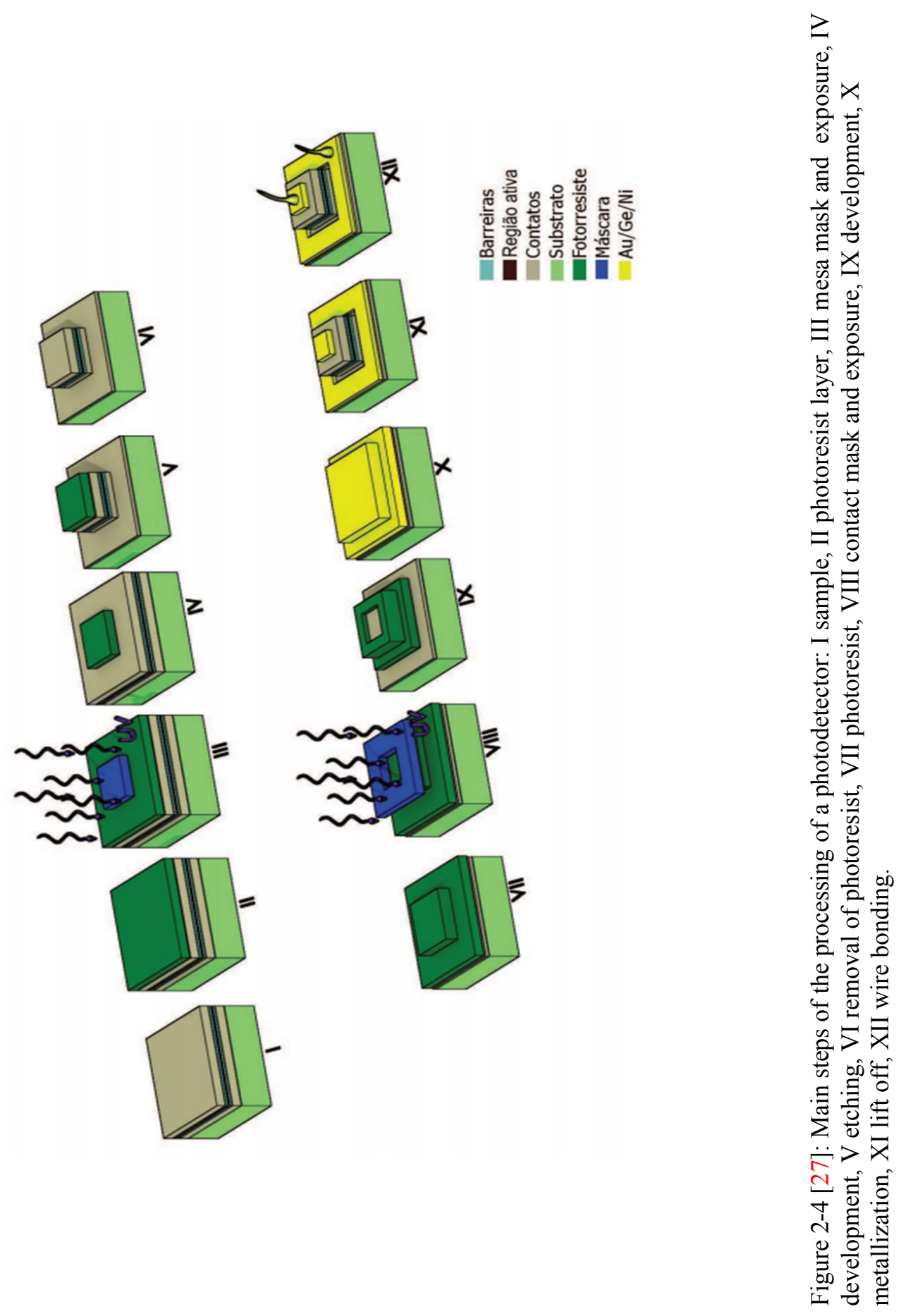




\section{2-2-2 Metallization and packaging}

Good Ohmic contacts on GaAs are usually obtained by the deposition of thin metallic films of $\mathrm{Ni}, \mathrm{Ge}$ and $\mathrm{Au}$. These metals were deposited sequentially using an electron-beam evaporator (figure 2-4-X) and a respective thickness of $25 \mathrm{~nm}, 50 \mathrm{~nm}$ and $150 \mathrm{~nm}$ for each of them. The sample was then soaked in acetone to dissolve the photoresist pattern and remove the excess of metal outside the small windows (this step is called lift off) that were defined in the last lithography step. At the end of the process, all the mesas will have a small top contact and a common bottom contact in between all the mesas (figure 2-4-XI).

To avoid the formation of a Schottky barrier that generally appears when a metal is directly deposited on top of a semiconductor, the sample was annealed (RTA, rapid thermal annealing) at $520^{\circ} \mathrm{C}$ during $30 \mathrm{~s}$ in order to get good Ohmic contacts.

Using a probe station connected to a semiconductor parameter analyzer, the quality of each device was quickly checked in order to decide which ones would be measured in more details.

Finally, the sample was fixed in a commercial chip carrier (figure 2-5 - a) with a drop of liquid carbon paint in order to get a good thermal contact between the sample and the chip carrier. Then the best mesas were connected with thin $\mathrm{Au}$ wires (diameter $\boldsymbol{r}=\mathbf{2 5} \boldsymbol{\mu m}$ ) to the pads of the chip carrier using a wire bonder (figure 2-5-b).
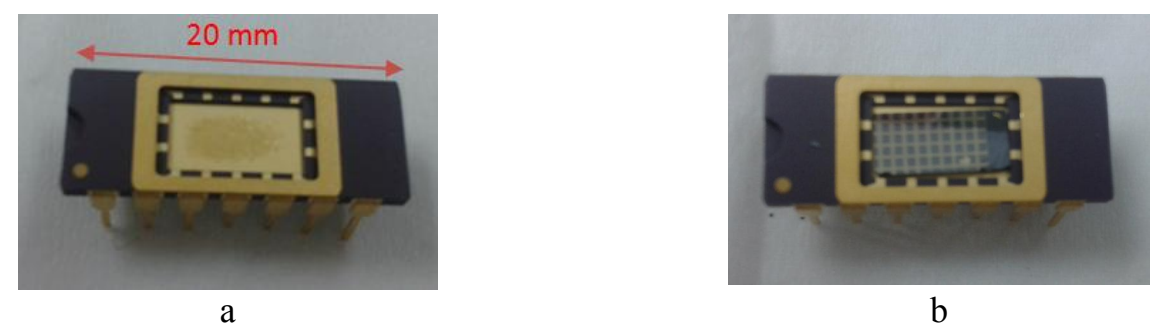

Figure 2-5: a) commercial chip carrier; b) Chip carrier with a sample fully processed. 


\section{Electro-optical characterization}

\section{2-3 Dark Current}

The dark current of a photodetector is the electrical signal that is measured in the device even without the presence of any external IR radiation (the device is kept in the dark). Since the dark current is generally several orders of magnitudes larger than the actual photocurrent of interest, it is an effect that limits the performance of the device and it is important to understand its physical mechanisms in order to keep it as low as possible and eventually to optimize the design of the structure. There are basically 3 sources of dark currents: thermally-excited electrons (figure 2-6-a) [7] that are normally the dominant component at temperatures above $30-50 \mathrm{~K}$, field effect tunneling (figure 2-6-b) that is relevant at high bias voltage (because of the strong deformation of the barrier profile), and direct tunneling through minibands that is important at low temperature and low bias voltage when the other mechanisms are weak (if the barriers are not too thick).

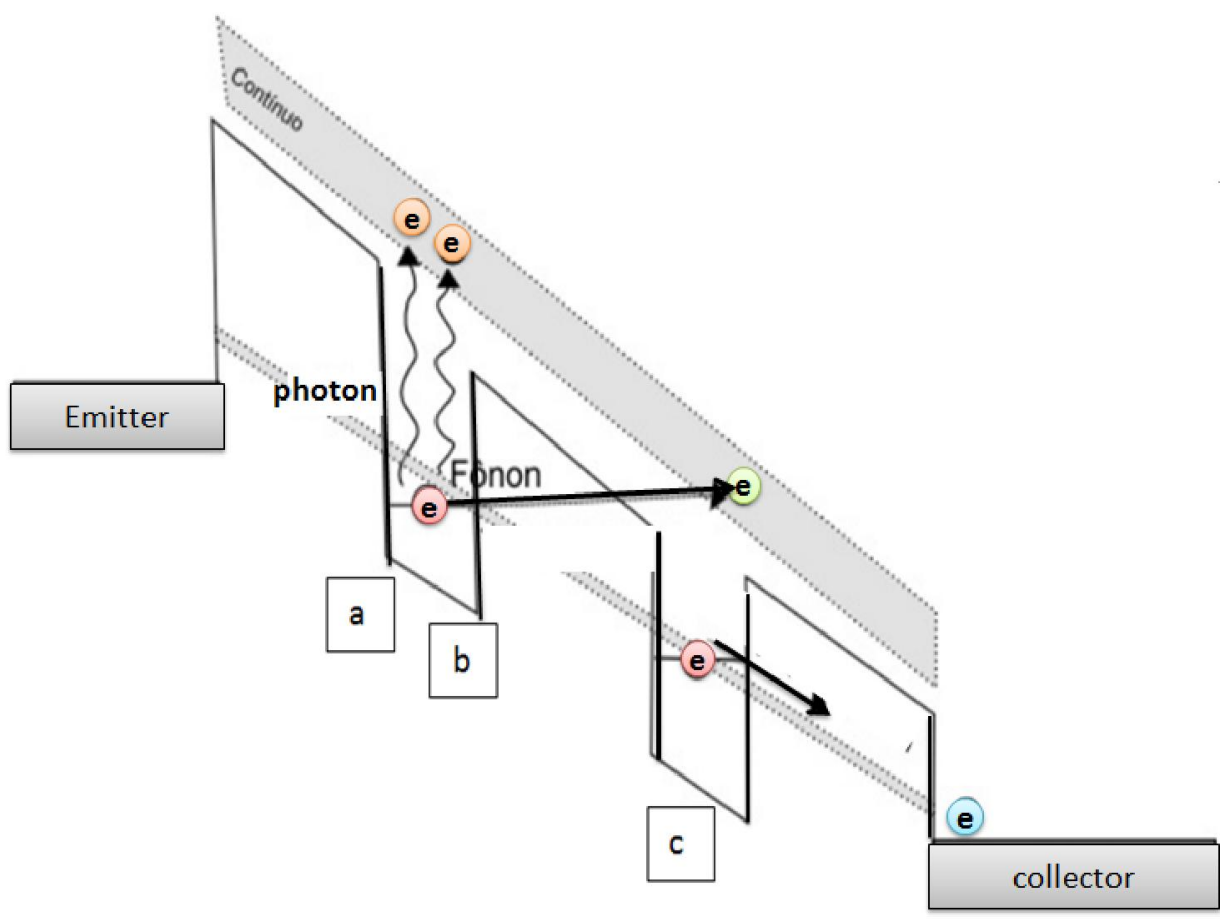

Figure 2-6: The main components of the dark current: a) thermally excited electrons above the barrier; b) field-effect tunneling; c) direct tunneling. 


\section{2-3-1 Experimental setup}

After processing the samples, the chip carrier containing the devices (figure 2-7-a) was plugged in the sample holder of a cold-finger cryostat (figure 27-b) and covered with a $\mathrm{Cu}$ spring plate to make a good thermal contact with the cold finger (figure 2-7-d). The benefits of the $\mathrm{Cu}$ spring plate will be shown in the next chapter. Then, the sample was covered with a copper shield to allow dark-current measurements (figure 2-7-e), acting also as a cold shield, and the whole cryostat (figure 2-7-f) was pumped down to $2.2 \times 10^{-5}$ Torr.

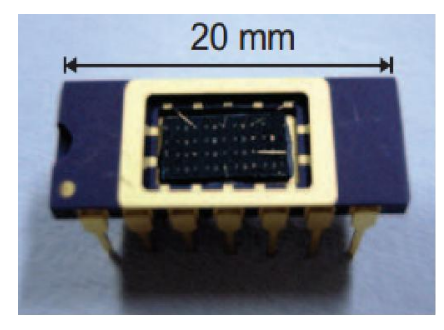

a

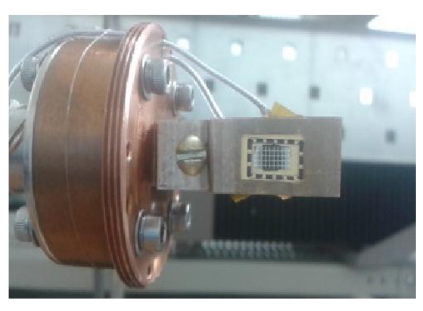

d

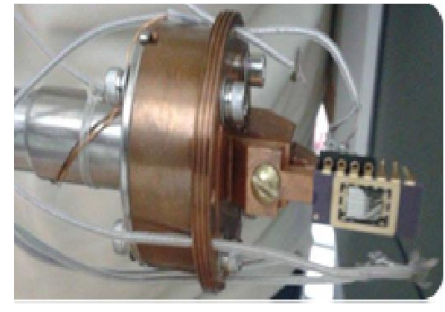

b

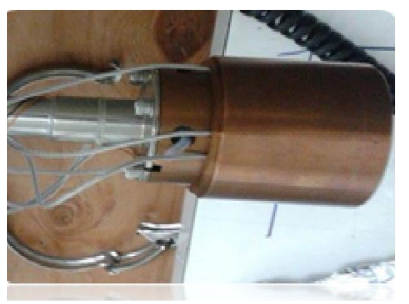

$\mathrm{e}$

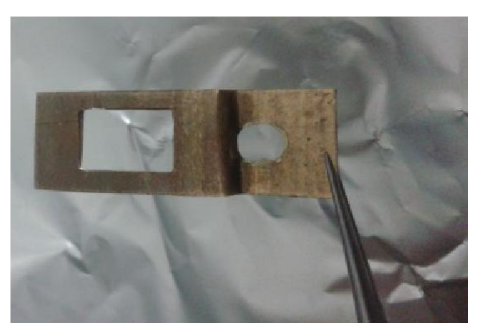

c

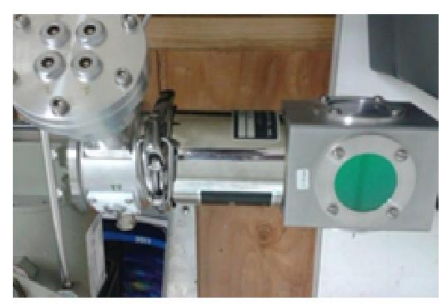

$\mathrm{f}$

Figure 2-7: a) Chip carrier containing the devices; b) chip carrier installed in the sample holder of the cryostat; c) $\mathrm{Cu}$ spring plate; d) $\mathrm{Cu}$ spring plate covering the chip carrier; e) dark shield surrounding the sample; f) cryostat fully mounted with Ge windows transparent in the IR spectrum (for further optical measurements).

The compressor of the Helium closed circuit was switched on to reach a temperature of the sample around $10 \mathrm{~K}$, and a heater located close to the sample was used to change the sample temperature between 10 and $300 \mathrm{~K}$ (using a LakeShore 325 temperature controller). The I-V curves were acquired by a sub-femto source-measure unit (Keithley 6430) remotely controlled by a computer. The whole experiment was controlled by a home-made software developed with LabView, as shown in figure 2-8. 


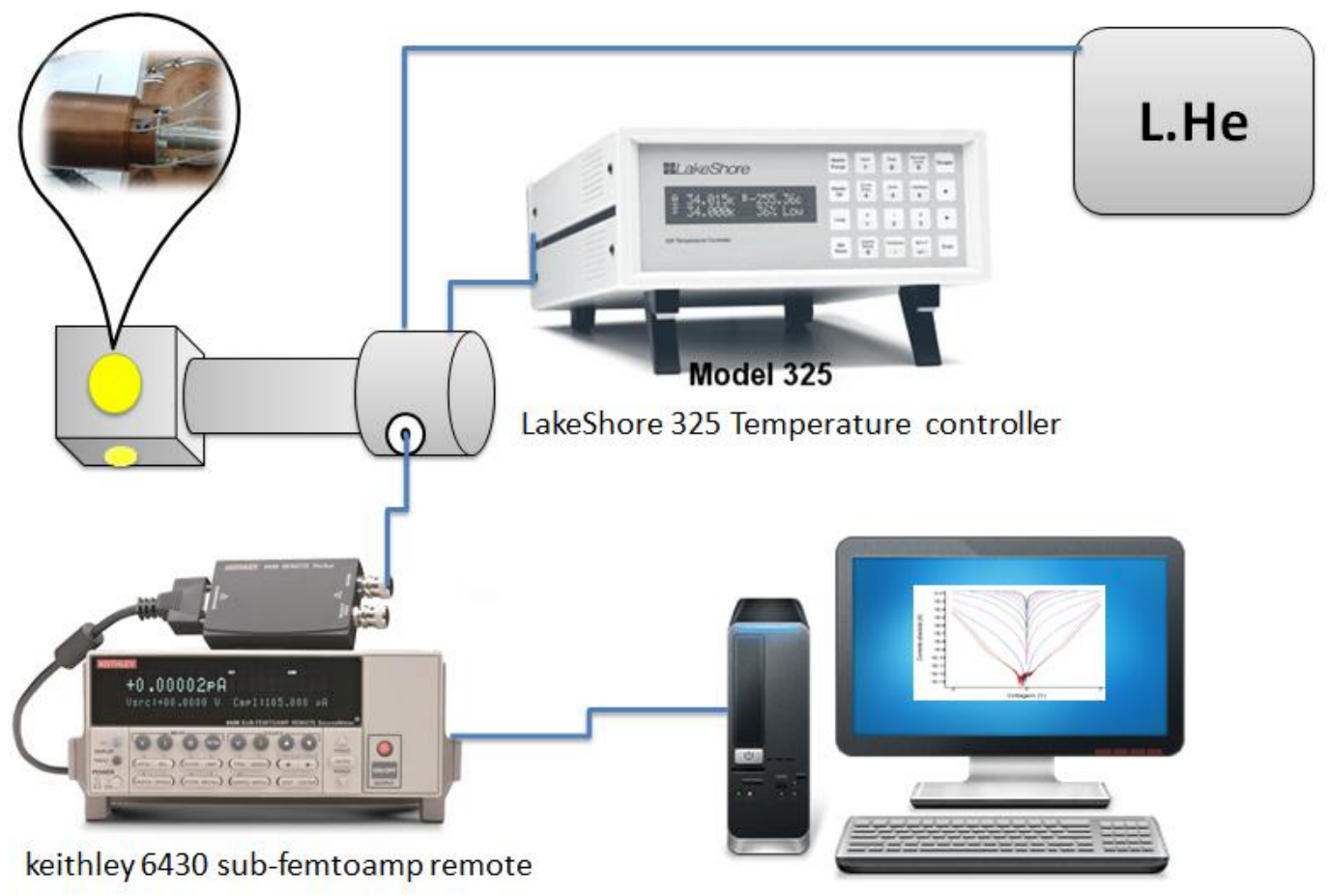

Figure 2-8: Experimental setup for I-V experiment (dark current).

\section{2-3-2 BLIP temperature}

The BLIP (background-limited infrared photodetector) temperature at a certain bias voltage is defined as the operating temperature at which the dark current is equal to the background photocurrent of a $300 \mathrm{~K}$ scene. This is generally obtained by wrapping the optical window with a cloth and acquiring an I-V curve without the dark shield. The total generation rate of carriers in an IR detector is the sum of the optical and thermal generation (equation 2-1) [28,29]:

$$
G=G_{t h}+G_{o p} \ldots \ldots \ldots \ldots(2-1)
$$

In an ideal case, the thermal generation should be much smaller than the optical generation which can be due to the background or to the signal of interest.

Since in general the background radiation is stronger that the signal itself, the detector operates in BLIP conditions when [30].

$$
\frac{\eta \Phi_{B} \tau}{t}>n_{t h} \ldots \ldots \ldots \ldots(2-2)
$$


where $n_{t h}$ is the density of thermal carriers at the temperature $T, \Phi_{B}$ is the total background photon flux density (photon $\mathrm{cm}^{-2} \mathrm{~s}^{-1}$ ) reaching the detector, $\tau$ is the carrier lifetime, and $t$ is the detector's thickness.

\section{2-4 Noise measurements}

The performance of a device is frequently determined by the signal-tonoise ratio. In a QDIP, the total current flowing in the device is the sum of the undesired dark current and of the photocurrent which is the signal of interest (the current that is generated by the electrons that are photoexcited by the infrared radiation hitting the surface of the device). In most intersubband photodetectors, the dark current is generally larger than the photocurrent, and therefore the noise mostly comes from the fluctuations of the dark current. In such devices, the noise can be divided into several components like 1/f noise, Shot noise, Johnson-Nyquist noise (also called thermal noise) and generation-recombination noise (also called GR noise). In general, in a QDIP, the first 2 types of noise are smaller than the thermal and GR noise and therefore are neglected. The 1/f noise is only relevant at very low frequencies (its amplitude is proportional to 1/f), while the Shot noise, which is due to the discrete nature of the electric charge, is the lowest of all the noise sources considered here and is generally important when a only a few events are considered (extremely low photon rates)

The thermal noise is present in any resistive element and is due to the thermal agitation of the charge carriers (fluctuations of the velocity vector). The amplitude $i_{t h}$ of the thermal noise of the current is given by equation 2-3 [28]:

$$
i_{\text {th }}^{2}=\frac{4 k T \Delta f}{R} \ldots \ldots \ldots \ldots(2-3)
$$

where $k$ is Boltzmann's constant, $\Delta f$ is the bandwidth that is used to measure the noise, $\mathrm{R}$ is the resistance of the detector, and $T$ is the absolute temperature.

The GR noise comes from the fact that charge carriers can be generated and can recombine randomly, thus leading to fluctuations of the total current. The amplitude $i_{G R}$ of the GR noise (in our case, mainly due to the dark current) is given by equation 2-4 [31]:

$$
i_{G R}^{2}=4 e g_{n} I_{d a r k} \Delta f \ldots \ldots \ldots \ldots(2-4)
$$


where $e$ is the electron charge, $g_{n}$ is the noise photoconductive gain (generally defined as the ratio of the recombination lifetime and the transit time of the carriers in the device), $I_{\text {dark }}$ is the dark current, and $\Delta f$ is the measurement band width. Since we can measure the noise of a device either as a voltage or as a current, it is generally called noise voltage or noise current, respectively. In this work, we will only use noise currents, as our main physical parameters are currents (photocurrent and dark current). The total noise current $i_{n}$ measured in a QDIP has both thermal and GR noise contributions [27] and is thus given by:

$$
i_{n}^{2}=i_{G R}^{2}+i_{\text {th }}^{2}=4 e g_{n} I_{\text {dark }} \Delta f+\frac{4 K T \Delta f}{R} \ldots \ldots \ldots \ldots(2-5)
$$

\section{2-4-1 Experimental setup}

The noise-current measurements were carried out with a dynamic signal analyzer and a current amplifier (figure 2-9). Since, in real operating conditions, the photodetector is always looking at a $300 \mathrm{~K}$ scene, the noisecurrent measurements should normally be performed in such conditions to determine the "real world" conditions. However, in the literature, the noisecurrent measurements are generally performed in the dark in order to limit the interference of external factors, and the same will be done here, using the dark shield around the devices.

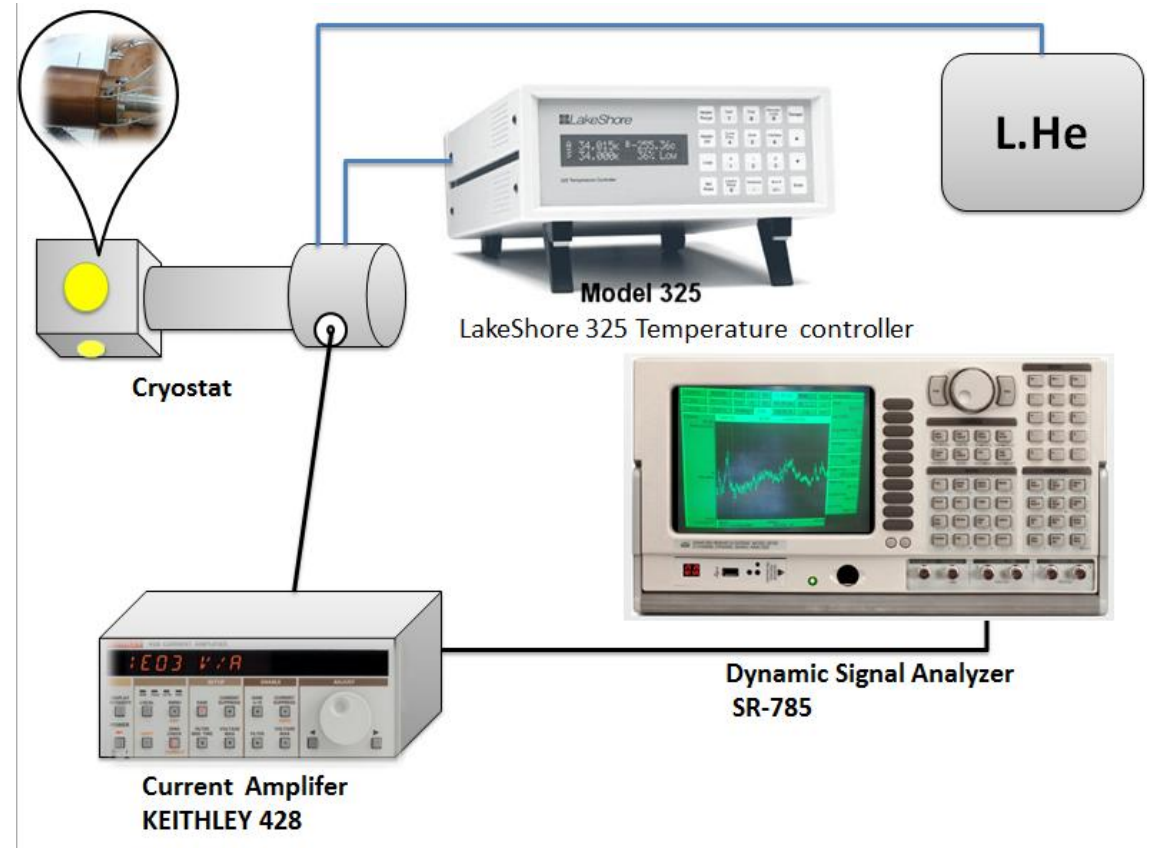

Figure 2-9: Experimental setup for the noise-current experiment. 
The current amplifier was used to apply a bias voltage on the device (generally from -2 to $2 \mathrm{~V}$ ), and a transimpedance gain as high as possible (between $10^{3}$ and $10^{8} \mathrm{~V} / \mathrm{A}$ ) should be selected to get more accurate measurement. The output of the amplifier provides thus a signal (voltage) proportional to the the real noise which is a function of the bias voltage and resistance of the device (equation 2-6) [32]

$$
V_{\text {noise }}=V_{\text {bias }} \times\left(1+\frac{G}{R_{S}}\right) \ldots \ldots \ldots \ldots \ldots \ldots(2-6)
$$

where $R_{S}$ is the resistance of the sample, $G$ is the gain of the current amplifier (in V/A), and $V_{\text {noise }}$ is the output noise voltage which will be analyzed by the dynamic signal analyzer. As a matter of fact, it is better to configure the spectrum analyzer to measure the spectral density of the noise, instead of the noise itself, because this parameter will be more useful in order to compute later the specific detectivity. The spectral density of the noise can be obtained automatically by the analyzer and is the square of the actual noise amplitude divided by the bandwidth $\Delta f$ used in the noise measurements [33].

We can see from equation 2-6 that the noise voltage increases as the value of the resistance of the sample decreases and/or the bias voltage increases, as can be observed in figure 2-10 which shows the spectral density of the noise voltage at two temperatures. At $10 \mathrm{~K}$, the noise of the device is very low and the spectrum is generally dominated by the eletromagnetic noise coming from the He cooling system. At higher temperature $(100 \mathrm{~K})$, the dark current is much higher and so is the noise of the device that can now be easily measured in a flat part of the spectrum (far from the $1 / \mathrm{f}$ noise). The real noise current was calculated using equation 2-7 [27] :

$$
I_{\text {noise }}=V_{\text {noise }} / G \ldots \ldots \ldots \ldots(2-7)
$$

In the case of figure 2-10-a, the spectrum analyzer indicates a noise voltage $V_{\text {noise }}=5.429 \mu \mathrm{V} / \sqrt{\mathrm{Hz}}$ at $212 \mathrm{~Hz}$ (square root of the noise spectral density), which means that the actual noise current is $I_{\text {noise }}=5.429 \times$ $10^{-14} \mathrm{~A} / \sqrt{\mathrm{Hz}}$. 


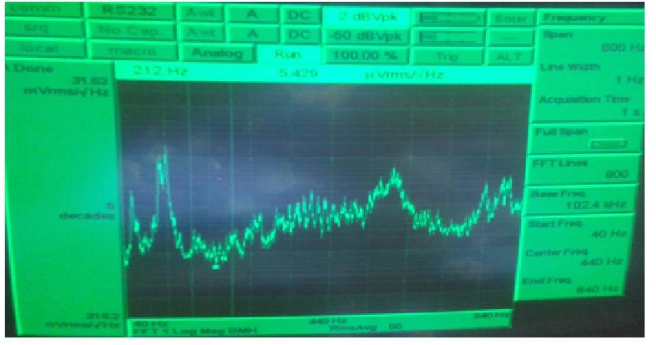

\begin{tabular}{|l|l|}
\hline Temperature & $10 \mathrm{~K}$ \\
\hline Bias voltage & $0,6 \mathrm{~V}$ \\
\hline Gain & $10 \mathrm{E} 8 \mathrm{~V} / \mathrm{A}$ \\
\hline Noise current & $5,429 \mathrm{E}-14 \mathrm{~A} / \sqrt{\mathrm{Hz}}$ \\
\hline
\end{tabular}

(a)

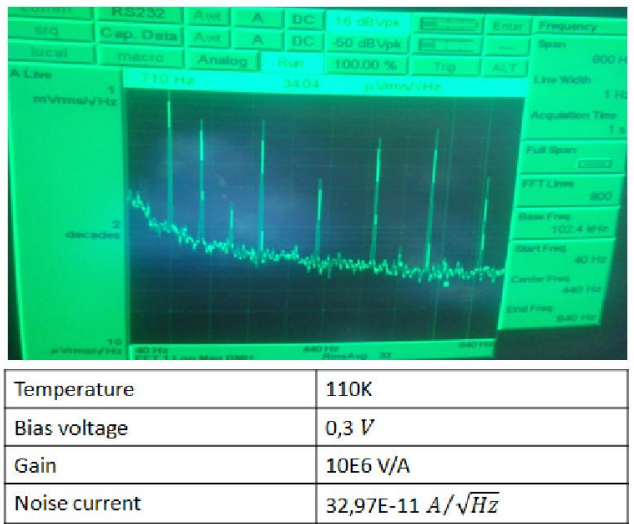

(b)

Figure 2-10: Spectral density of the noise voltage of a device at two different temperatures.

\section{2-5 Photocurrent with black body and responsivity experiment}

The responsivity measurement aims to determine the efficiency of the device by comparing its electrical output (photocurrent) and optical input (the number and energy of the photons hitting the device per unit of time). The photocurrent itself is generally measured using lock-in techniques that allow automatically the subtraction of the dark current from the total current, leaving then only the real photocurrent generated by the infrared radiation hitting the detector. Since the optical input needs to be known accurately, the best way to generate the infrared radiation is by using a calibrated black body. In such conditions, the input optical power can be calculated with the following equation [45]:

$$
P=\int_{\lambda_{1}}^{\lambda_{2}} T_{J C} \cdot \varepsilon_{b b} . \Omega \cdot A_{d} \cdot L(\lambda, T) \cdot d \lambda \ldots \ldots \ldots \ldots(2-8)
$$

where $\lambda_{1}$ and $\lambda_{2}$ are the integration limits determined by the own optical absorption spectrum of the device, $T_{J C}$ is transmission of cryostat's window , $\varepsilon_{b b}$ is the emissivity of black body, $A_{d}$ is the detector area, $\Omega$ is solid angle and it is given by $\Omega=\frac{(d / 2)^{2}}{D^{2}+(d / 2)^{2}}$ where $\mathrm{D}$ is the distance between the sample and the exit of the black body, $\mathrm{d}$ is $R_{b}$ is aperture diameter, and $L(\lambda, T)$ is the spectral emittance of the black body (from Planck's equation). The experimental setup for the photocurrent measurements is schematically shown in figure 2-11. 

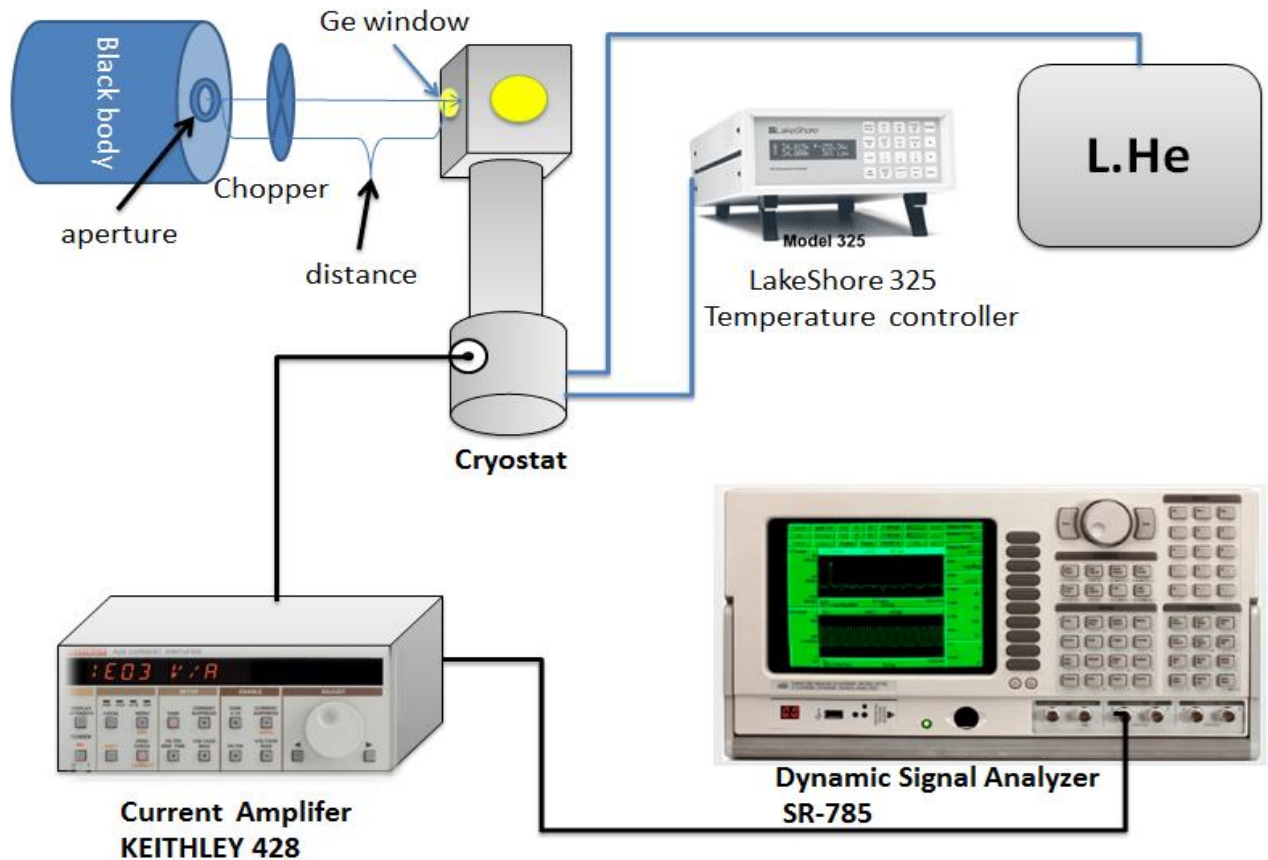

Figure 2-11: The experimental set up for the photocurrent / responsivity measurements.

The infrared radiation from the black-body source is directed through the chopper and Germanium window onto the detector mounted inside the cryostat. The dynamic signal analyzer performs a real-time Fast Fourier Transform (FFT) of the total current flowing in the device and shows the amplitudes of all the frequencies present in the signal, as illustrated in figure 2-12.

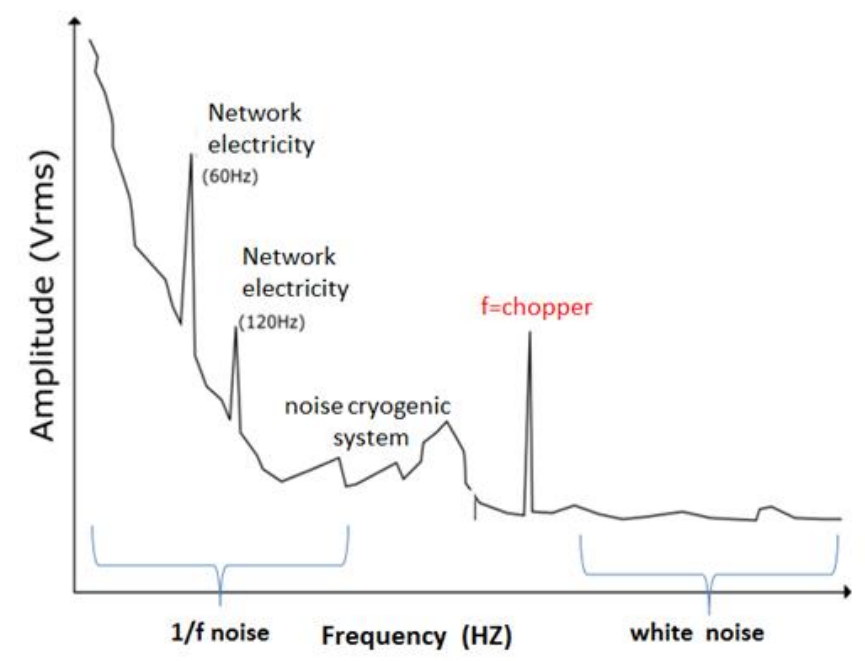

Figure 2-12: Typical frequency spectrum of the total current obtained by the dynamic signal analyzer, showing the $1 / \mathrm{f}$ noise, the harmonics of the network, the noise from the cryogenic system, the signal of the photocurrent, and the noise from the device (white noise). 
The advantage of this kind of setup over a conventional lock-in technique is that the photocurrent can be measured easily by checking directly the intensity of the signal at the exact frequency of the chopper, but the background of the noise can also be simultaneously assessed in order to monitor the evolution of the device's performance. The responsivity is thus given by equation 2-9 [34, 35]

$$
R=\frac{I}{P} \quad \ldots \ldots \ldots \ldots(2-9)
$$

where $I$ is the photocurrent (reading of the analyzer divided by the gain of the transimpedance amplifier) and $P$ is the input optical power given by equation $2-8$.

\section{2-6 Spectral response (absorption measurements)}

Absorption measurements are necessary in order to determine the wavelength range in which the device will operate. Fourier-transform infrared (FTIR) spectroscopy is used to investigate the optical response of the photodetector. In this case, the standard detector of the FTIR system is substituted by our photodetector in order to investigate its properties (figure 2-13), but the internal radiation source and electronics are used to illuminate the sample and process the data normally. As always for this technique, a background spectrum must be provided and was obtained by simply shuttering the infrared radiation, keeping all the other experimental parameters constant.

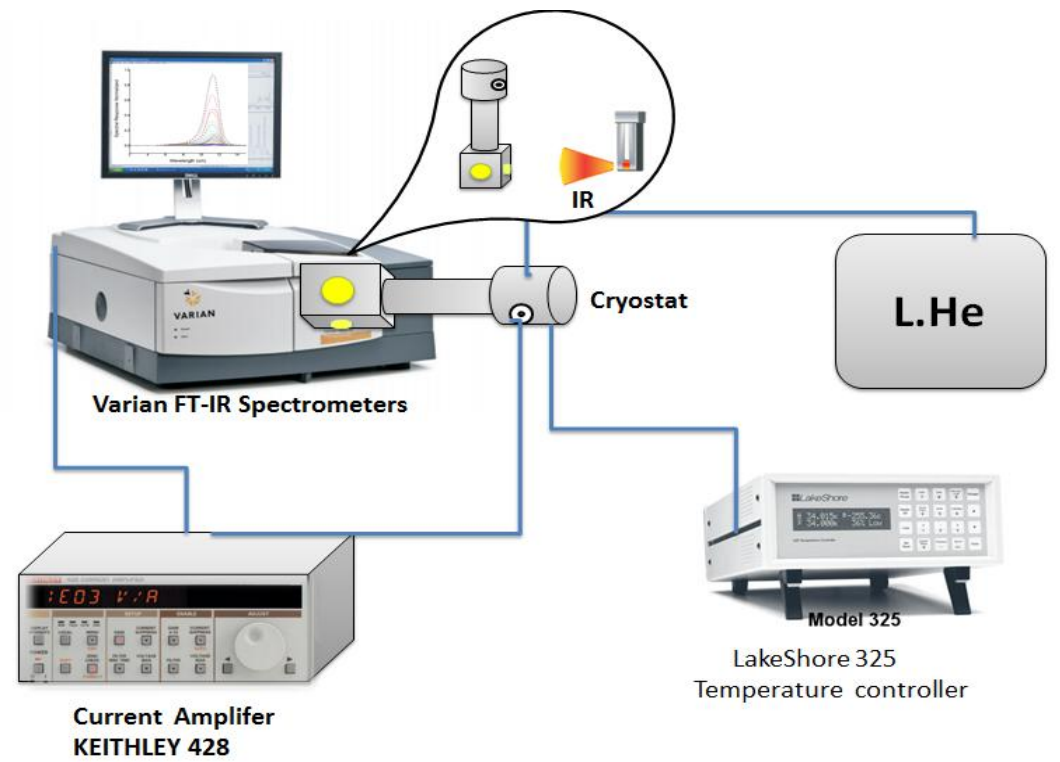

Figure 2-13: Experimental setup for absorption measurements using FTIR spectroscopy. 
These absorption measurements are also important for the calculation of the absolute responsivity, because the width of the absorption spectrum at half maximum (figure 2-14) [27] will be taken as the integration limits $\lambda_{1}$ and $\lambda_{2}$ needed to compute the optical power hitting the device (equation 2-8).

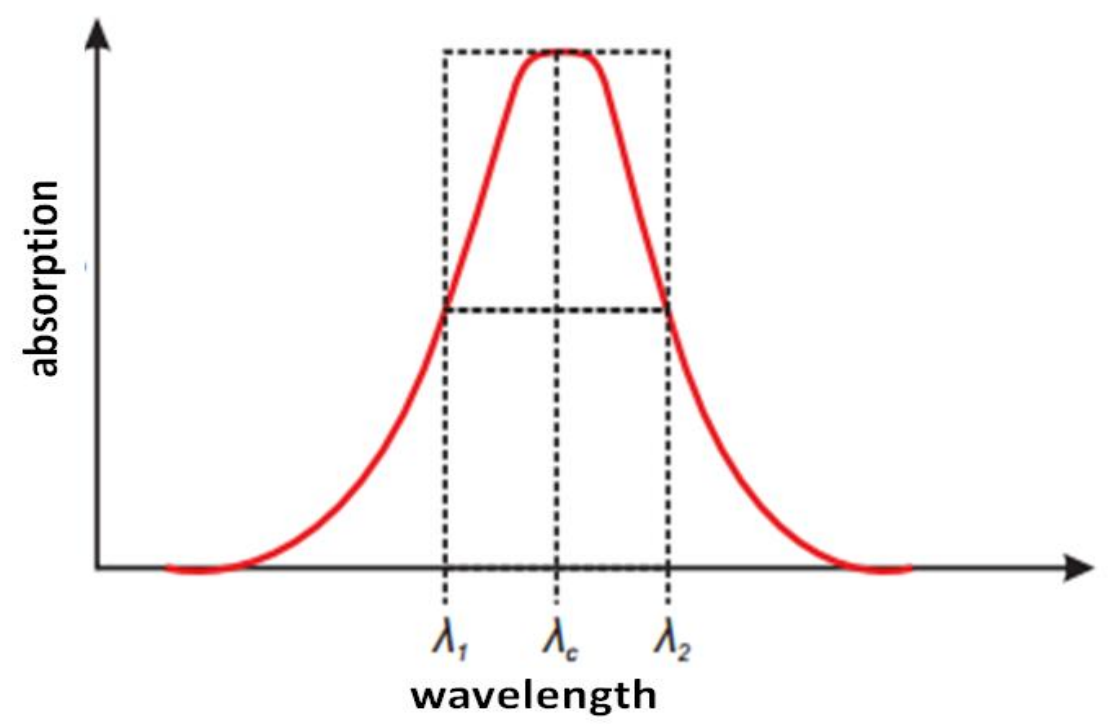

Figure 2-14: Width at half maximum (determined by $\lambda_{1}$ and $\lambda_{2}$ ) of a typical absorption spectrum of a photodetector. 


\section{Chapter 3: Photodetectors based on submonolayer quantum dots and on usual Stranski-Krastanov quantum dots.}

In this chapter, the experimental results of two infrared photodetectors will be presented. The first device was a standard QDIP containing conventional Stranski-Krastanov quantum dots (SK-QDs, sample \#3551) while the second one was based on submonolayer quantum dots (SMLQDs, sample \#3601).

\section{3-1 Design, growth \& processing of the samples}

Samples \#3551 and \#3601 were grown by a PhD student of our group on a semi insulting (100) GaAs substrate using the molecular beam epitaxy (MBE) system of the Laboratory of New Semiconductor Materials (LNMS) of the Institute of Physics - at USP - and processed in our laboratory as well using conventional photolithography, wet etching and metallization techniques. Sample \#3551 was grown in usual conditions for SK quantum dots. The InAs growth rate was close to $0.1 \mathrm{ML} / \mathrm{s}$ and the QDs started to nucleate on the surface after 1.7 ML, as could be seen on the RHEED screen. Each QD layer had a nominal thickness of 2.2 MLs, and the QD

areal density in these growth conditions was close to $4 \times 10^{10} \mathrm{~cm}^{-2}$. The Si shutter was kept open during the growth of the InAs material in order to get 2 electrons in the ground state of each QD. Sample \#3601 had a structure similar to sample \#3551, but the SK-QDs were replaced by SMLQDs. However, since the growth of SML-QDs is more complex than the growth of SK-QDs, only 10 layers of SML-QDs were grown in sample \#3601 (instead of 30 in sample \#3551). Figure 3-1 shows their structure. 


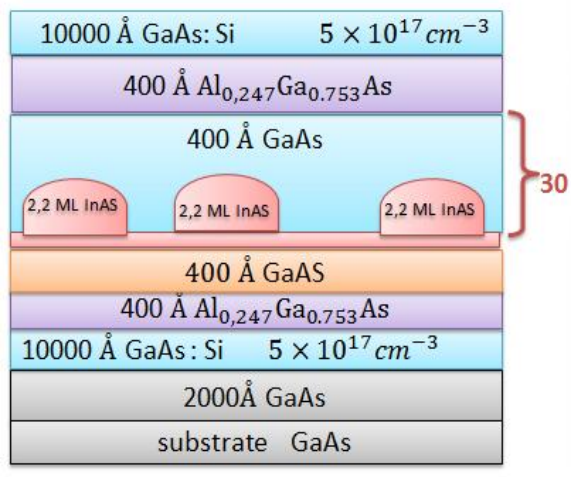

Sample SK-QD \# 3551

a

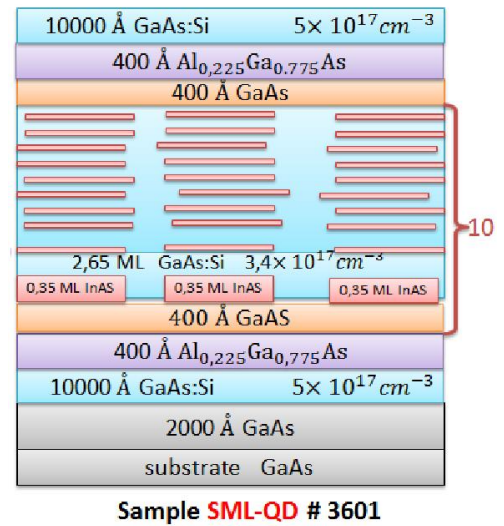

b

Figure 3-1: a) Structure of sample \#3551 (containing conventional SK-QDs; b) of sample \#3601 (containing SML-QDs).

The first step was to be sure that the growth of the QDIP containing SMLQDs was successfully carried out. Therefore, a similar sample was grown, without the 2 thick Si-doped contact layers, in order to check with photoluminescence (PL) measurements if the signal related to the SMLQDs was present. Indeed, case the growth was performed in the wrong conditions, the InAs islands would not be formed and the sample behave as a $\mathrm{In}_{0.12} \mathrm{Ga} 0.88 \mathrm{As} / \mathrm{GaAs}$ quantum well having a thickness of $85 \mathrm{~nm}$ $((0.35+2.65) \times 10 \mathrm{MLs})$. The PL spectrum of the sample is shown in figure $3-2 \mathrm{a}$ at $77 \mathrm{~K}$. At low temperature, it is very similar to the PL spectrum of an $\mathrm{In}_{0.12} \mathrm{Ga} 0{ }_{88} \mathrm{As} / \mathrm{GaAs}$ quantum well (not shown here) and therefore they are difficult to distinguish. It is worthwhile mentioning here that the PL spectrum of figure $3-2 \mathrm{a}$ is much narrower $(11 \mathrm{meV})$ than the one of common SK-QDs that usually have a full width at half maximum (FWHM) between 50 and $80 \mathrm{meV}$, suggesting that these SML-QDs are more homogeneous than the SK-QDs. Figure 3-2b shows the PL spectrum of SML-QDs at $180 \mathrm{~K}$ and, as a reference, the spectrum of a similar sample that was grown intentionally in the $\mathrm{c} 4 \mathrm{x} 4$ surface reconstruction and that turned to be a InGaAs quantum well. It can be seen that, at higher temperature, both samples behave very differently: the signal of the quantum well is very broad and weak, and is typical of band to band optical recombinations due to the escape of the carriers from the quantum well [45] while the signal of the SML-QDs is much stronger and exciton like (narrow peak around $1.38 \mathrm{eV}$ ), confirming that the carriers are still confined by the strong potential of the SML-QDs, as expected. 


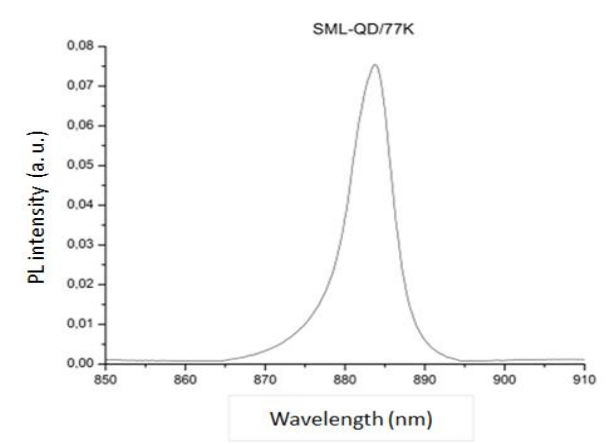

a

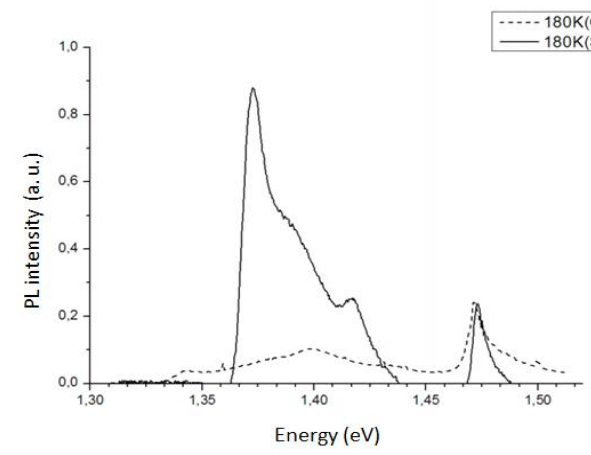

b

Figure 3-2: a) Photoluminescence spectrum of a sample of SML-QDs at $77 \mathrm{~K}$. b) PL spectra of a sample of SML-QDs at $180 \mathrm{~K}$ and of a InGaAs/GaAs quantum well with a similar composition and thickness [46].

\section{3-2 Electro-optical characterization}

Usually, we start the characterization of the devices by performing dark current measurements in order to have an idea of their performance. In this case, we were very surprised to detect a high dark current and, when other devices previously tested were checked again, we discovered that all of them had an abnormally large dark current as well. After analyzing these data and checking all the cables and the whole setup, we concluded that there was a bad thermal contact between the bottom part of the chip carrier and the cold finger of the cryostat, leading to a higher sample temperature than expected. The geometry of the sample holder was thus slightly changed and an extra spring plate (figure 3-3), in thermal contact with the cold finger, was installed in order to keep pushing the chip carrier into the socket and against the cold finger (using Apiezon $\mathrm{N}$ grease to improve the thermal contact).

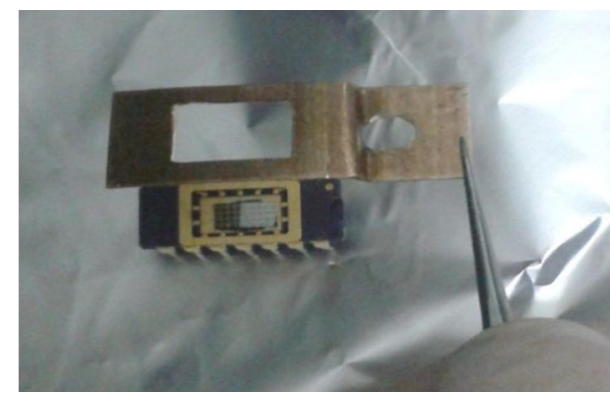

a

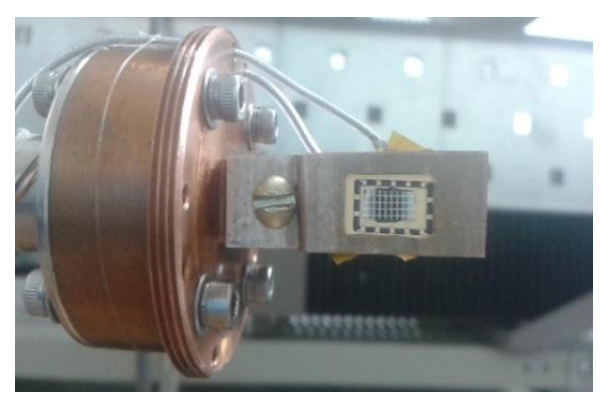

b

Figure 3-3: (a) $\mathrm{Cu}$ spring plate; (b) $\mathrm{Cu}$ spring plate installed on top of the chip carrier and pressing it against the cold finger. 
Figure 3-4 shows the dark-current (I-V) curves of a device (sample \#3691) with and without the $\mathrm{Cu}$ spring plate. It can be seen that, when the $\mathrm{Cu}$ spring plate is installed, the dark current is several orders of magnitude lower, which means that the sample temperature is lower and probably very close to the expected value measured by the thermocouple.

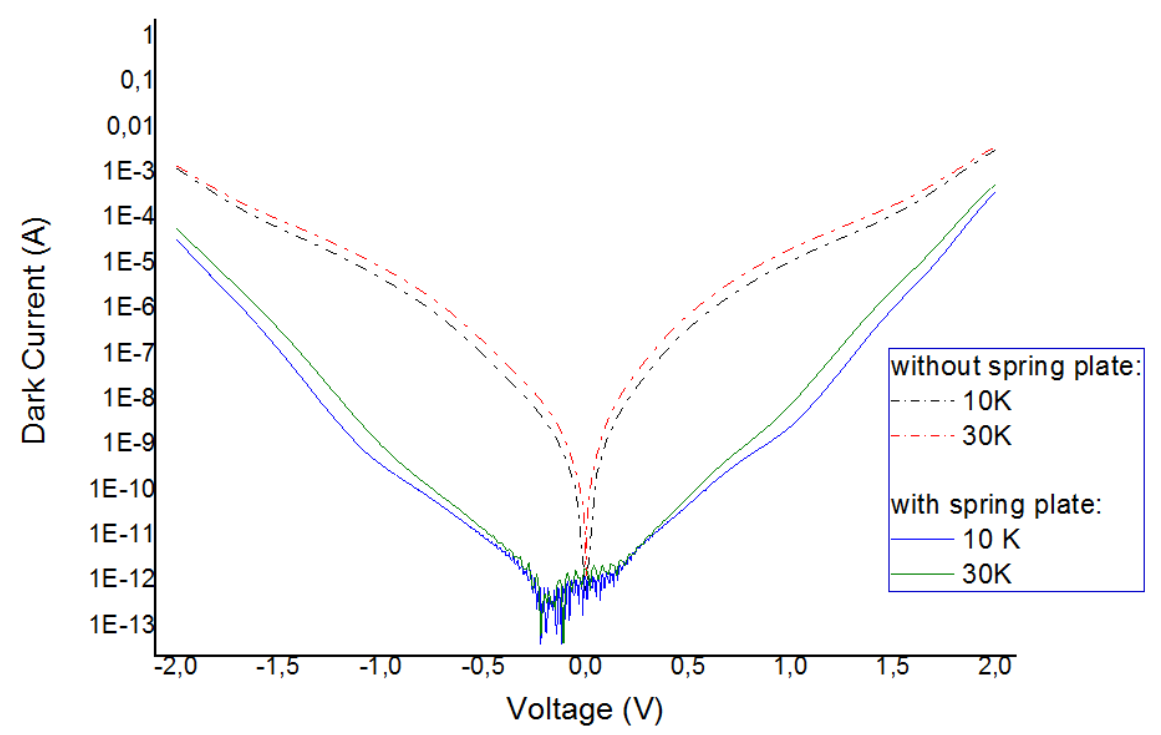

Figure 3-4: Dark-current curves of a device with (full lines) and without (dashed lines) the $\mathrm{Cu}$ spring plate.

\subsubsection{Dark current}

The experimental dark-current curves (I-V) of samples \#3551 and \#3601 are shown in figure 3-5. They show that, below $50 \mathrm{~K}$, the dark current curves are almost identical and don't dependent on temperature, meaning that it is probably due to direct tunneling through the barriers [36, 37], since the carriers have not enough thermal energy to access the upper levels. The current is very low at low bias and is limited by the detection floor of the experimental setup. At higher bias, more electrons are injected from the contact layers, which results in an increase of the Fermi level and a consequent decrease of the activation energy. In addition, the tops of the barriers are distorted by the electric field of the external bias, and the dark current starts to be dominated by field effect tunneling. At higher temperature, the dark current starts to increase rapidly with temperature as 
more and more electrons are thermally activated to the upper energy levels, as expected [38].

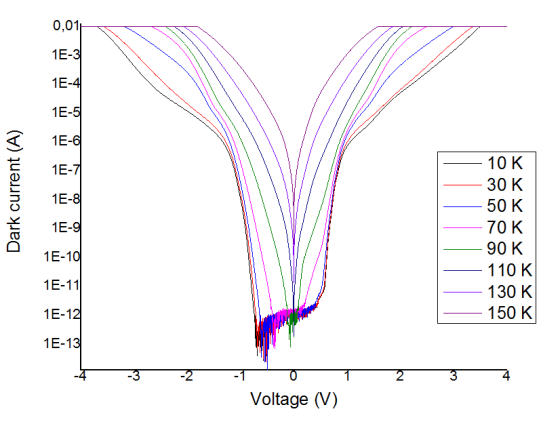

a

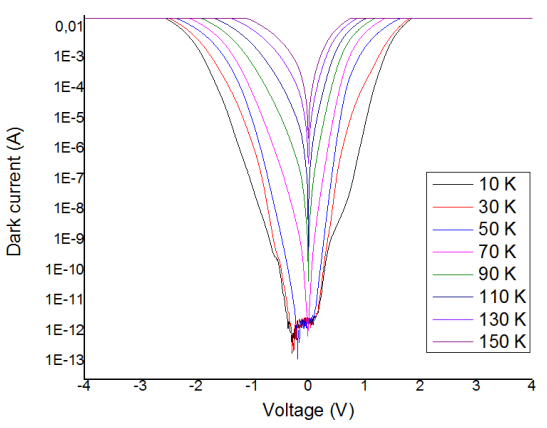

b

Figure 3-5: Dark current of sample \#3551 (a) and \#3601 (b).

In general, it can be seen that the dark current of the device containing SML-QDs (\#3601) is larger than the one of the device containing conventional QDs (\#3551), even at low temperature (figure 3-6), a fact that a priori might be due to the much higher density of nanostructures achieved by the SML technique.

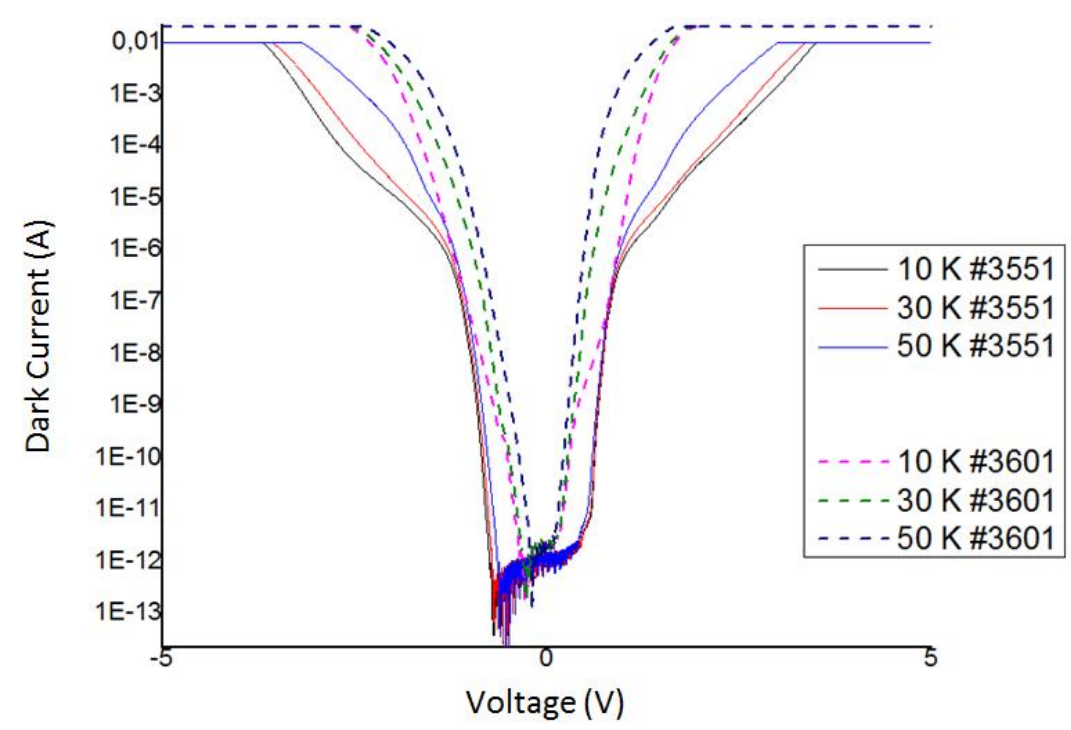

Figure 3-6: Dark current of sample \#3551 and sample \#3601 at low temperature.

An Arrhenius plot of the dark current of both samples as a function of temperature for a bias of $0.6 \mathrm{~V}$ is reported in figure 3-7. This bias $(0.6 \mathrm{~V})$ was chosen because, as will be shown later, the maximum detectivity value was obtained at that bias value for sample \#3551. 


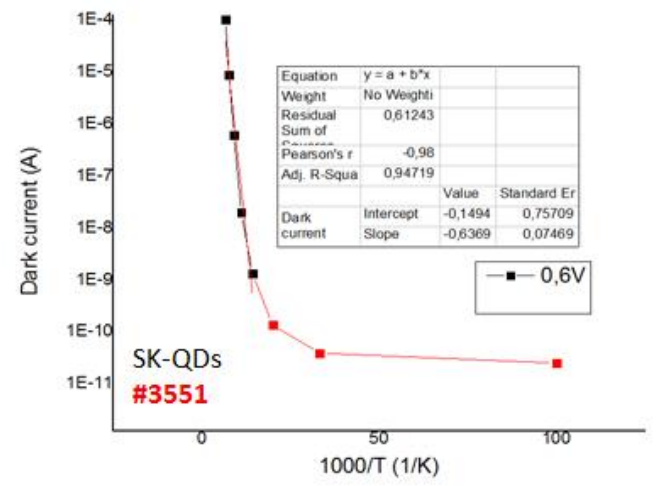

(a)

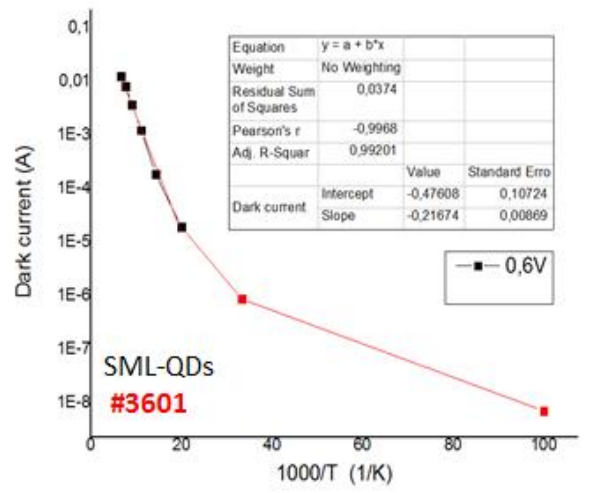

(b)

Figure 3-7: Dark current as a function of temperature at a bias of $0.6 V$ for sample \#3551 (a) and \#3601 (b).

The linear behavior of the curves at high temperature reflects the exponential increase of the dark current due to thermal excitation of the carriers above the barriers. At lower temperature (below $70 \mathrm{~K}$ for sample \#3551 and below $50 \mathrm{~K}$ for sample \#3601) the dark current is relatively insensitive to temperature as already observed in figure 3-5.

Based on Arrhenius's equation:

$$
I=I_{0} \times e^{-E_{\text {activ }} / k T} \ldots \ldots \ldots \ldots(3-1)
$$

we can write $\log (I)=\log \left(I_{0}\right)-\frac{\log (e) \times E_{\text {activ }}}{k} \frac{1}{T}$

Therefore, the slope of the curve in figure 3-7 will be given by:

$$
\text { Slope }=\frac{-\log (e) \times E_{\text {activ }}}{k} \ldots \ldots \ldots \ldots(3-2)
$$

where $I$ is the dark current, $I_{0}$ is a constant, $k$ is Boltzmann's constant, $T$ is the absolute temperature, and $E_{a c t i v}$ is the activation energy of the process.

The activation energy was found to be $126 \mathrm{meV}$ for sample \#3551 and 42,8 meV for sample \#3601. As shown in figure 3-8 [6], the activation energy is actually the difference between the Fermi energy of the structure and the energy of the top of the barriers. Therefore, the lower activation energy in sample \#3601 might also explain why the dark current is larger for that sample. Indeed, it could eventually come from a high doping level that was originally based on the density of small 2D islands reported in the literature and that might have been overestimated for our specific case (since it was not optimized). Another possible (and more probable) reason 
for a lower activation energy is that the energy levels of the SML-QDs might be higher than in the case of SK-QDs [39]. Although the energy levels in SK-QDs are still somewhat difficult to predict as a consequence of the lack of information about their actual size, composition and geometry, some recent calculations taking account of the strain and In segregation (together with microscopy and PL experimental data) were able to provide more reliable results $[40,41]$. SML-QDs face the same problems, but they are smaller and more difficult to evidence than usual SK-QDs, and there are only a few works available in the literature [44]. As already mentioned in chapter 2, and can be seen in figure 3-1, the SML-QDs of sample \#3601 are taller and narrower than usual SK-QDs, but their average In content $(12 \%)$ is also lower, a factor that would be enough to shift their energy levels to higher values and therefore decrease their activation energy.

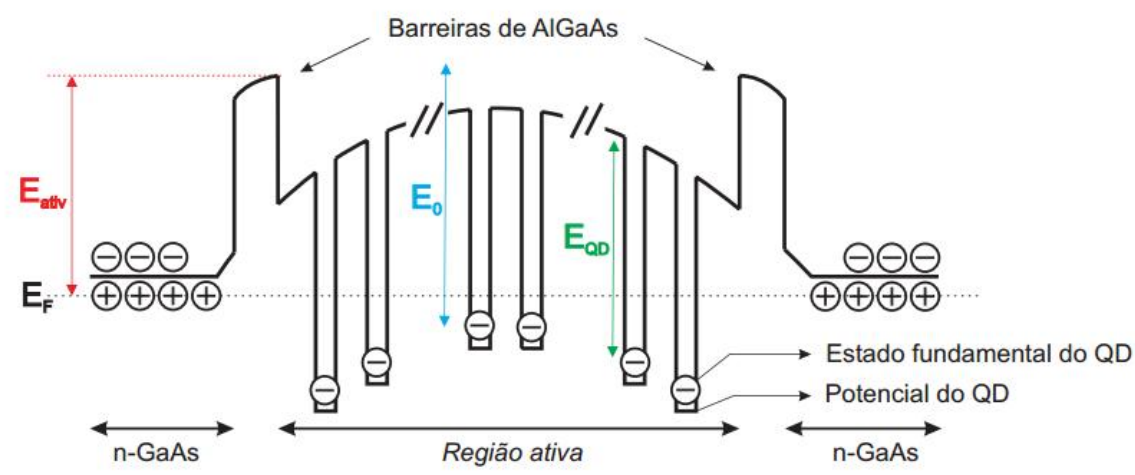

Figure 3-8: Band structure of a QDIP showing the activation energy, which is the difference of energy between the Fermi level $\mathrm{E}_{\mathrm{F}}$ and the top of the AlGaAs barriers. $\mathrm{E}_{0}$ is the theoretical energy required to excite an electron from the ground state of the quantum dot to the top of the AlGaAs barriers, and $\mathrm{E}_{\mathrm{QD}}$ is the calculated ground-state energy of the QDs.

\subsubsection{Noise current}

Figures 3-9 shows the noise current of both samples. We can notice that the noise reflects the behavior of the dark current: below $50 \mathrm{~K}$, the noise is generally very low (reaching the floor of the experimental setup) and almost independent of temperature, while, at higher temperature, it starts to increase rapidly. We can also observe that the noise of sample \#3551 is lower than the one of sample $\# 3601$, as expected from their respective dark current. 


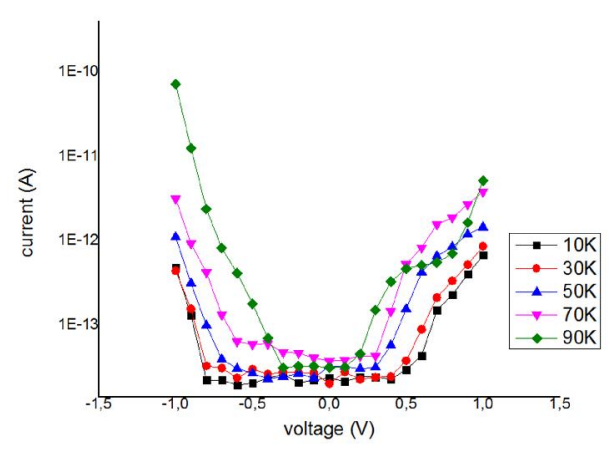

a

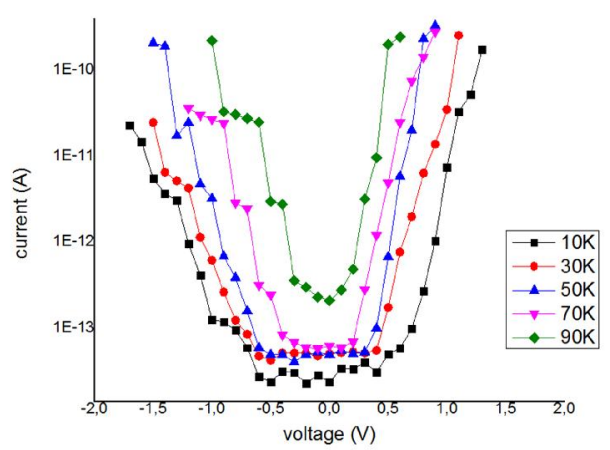

$\mathrm{b}$

Figure 3-9: Noise-current measurements as a function of bias voltage and temperature (a) for sample \# 3551 and (b) for sample \#3601.

Using equation 2-3, we can calculate the intensity of the thermal noise of sample \# 3551 at low bias (for example $0.1 \mathrm{~V}$ ) and low temperature $(10 \mathrm{~K})$ using a bandwidth $\Delta f=1 \mathrm{~Hz}$ and $i_{t h}=0.487 \times 10^{-14} \mathrm{~A} / \sqrt{\mathrm{Hz}}$. Since that value is close to the one measured in figure 3-9 for the same parameters $\left(2.144 \times 10^{-14} \mathrm{~A} / \sqrt{\mathrm{Hz}}\right)$, it means that the thermal noise is significant at low bias and probably dominates the other components like $1 / \mathrm{f}$ noise and GR noise.

As the bias increases (above $0.7 \mathrm{~V}$ for sample \#3551 and $0.9 \mathrm{~V}$ for sample \#3601) or the temperature is raised, the measured noise increases much faster than the thermal noise, because the GR noise (equation 2-4) kicks in due to field-effect tunneling and thermal excitation.

\subsubsection{Photocurrent with a black body}

The experimental setup used to simultaneously measure the noise (with the FFT spectrum analyzer) and photocurrent (with a black body) is shown in figure 2-11. In our experiment, the temperature of the black body was set to $T=800{ }^{\circ} \mathrm{C}$, its aperture size was $R=12,7 \mathrm{~mm}$, and the distance between the device and the black body was $D=21 \mathrm{~cm}$. These geometrical parameters of the setup will be necessary to calculate the incident optical power (equation 2-8) needed to compute the responsivity and, later, the detectivity. A Germanium window with an anti-reflective coating was used in order to allow a maximum transmission in the $3-12 \mu \mathrm{m}$ range and to absorb photons which have a wavelength lower than $3 \mu \mathrm{m}$ to avoid possible interband transitions that might interfere with the IR response of the device. The photocurrent measurements are reported in figure 3-10. As can be 
observed, the photocurrent is very similar at all temperatures, which is consistent with the fact that the doping was adequate (i.e. there are around 2 electrons in the ground state of each QD). Once again, the signal coming from the SML-QDs sample \#3601 is slightly larger, probably as a consequence of the larger density of dots with respect to the SK-QDs case (although there were less periods in the structure).

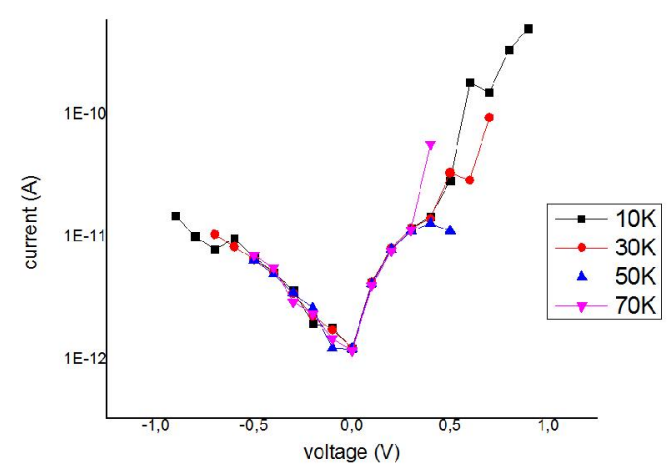

a

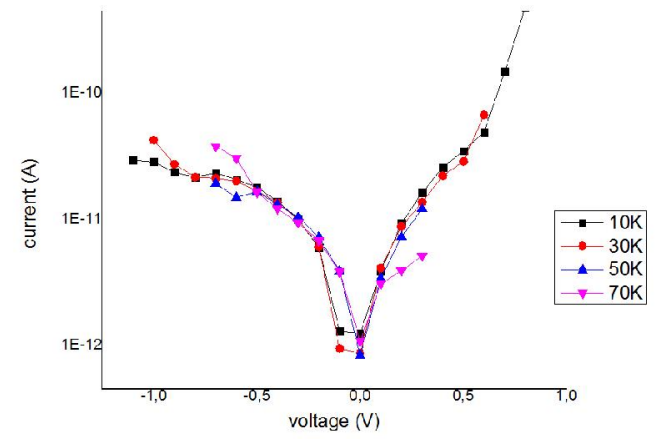

b

Figure 3-10: Photocurrent as a function of bias and temperature using a black body for (a) sample \#3551 and (b) sample \#3601.

\subsubsection{Spectral Response}

Fourier-transform infrared (FTIR) spectroscopy was used to investigate the optical spectral response of both samples, but we were unable to get a signal from sample \#3601 because of the saturation of the measurement system at any temperature and bias (probably due to the larger dark current and, consequently, larger noise). That didn't happen during the photocurrent measurement with the black body because, as mentioned in section 2-5, the signal was chopped and then fed into a spectrum analyzer that allows a more effective separation of the signal from the background noise (thanks to the FFT technique). The spectral response (normalized) of sample \#3551 at $10 \mathrm{~K}$ is reported in figure 3-11 for several bias values. 


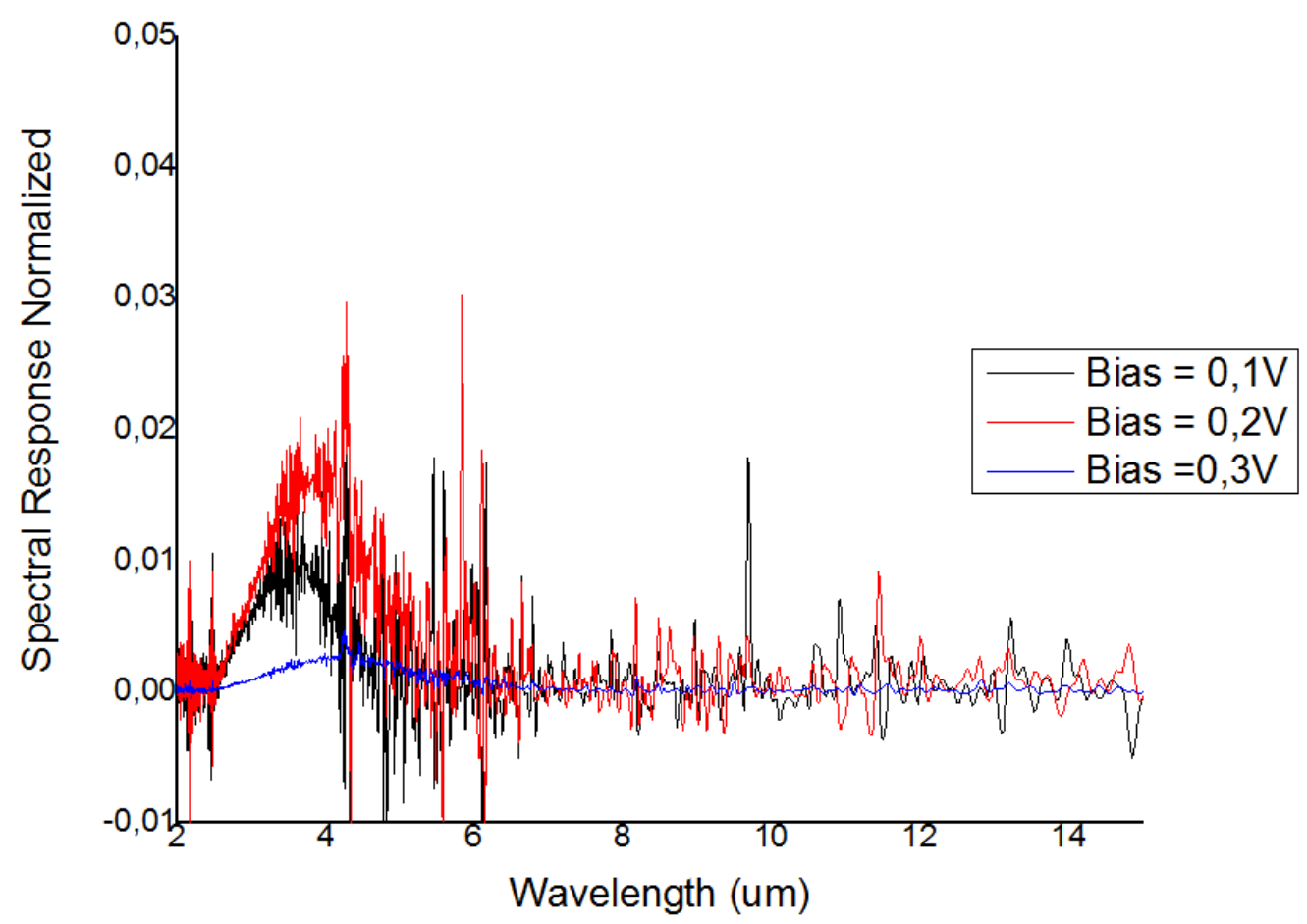

Figure 3-11: Spectral response (normalized) of sample \#3551 at $10 \mathrm{~K}$ for several bias voltages.

\section{3-2-5 Responsivity}

Once the photocurrent (measured with the black body) and the spectral response (measured by FTIR spectroscopy) were obtained, it was then possible to calculate the power of the radiation hitting the detector surface (for each bias at $10 K$ ) in order to calculate the responsivity which determines the conversion efficiency of the device by comparing its electrical output (photocurrent) and optical input (number and energy of the photons hitting the device per unit of time), as described by equation 2-9.

A small program written in Mathematica was used to calculate the optical power hitting the detector surface using all the geometrical and physical parameters involved in the experiment. The responsivity $R$ was then calculated from all the available parameters and the results are reported in figure 3-12 for sample \#3551 only, since we were not able to measure the absorption spectrum of sample \#3601 that was necessary to calculate the responsivity. 


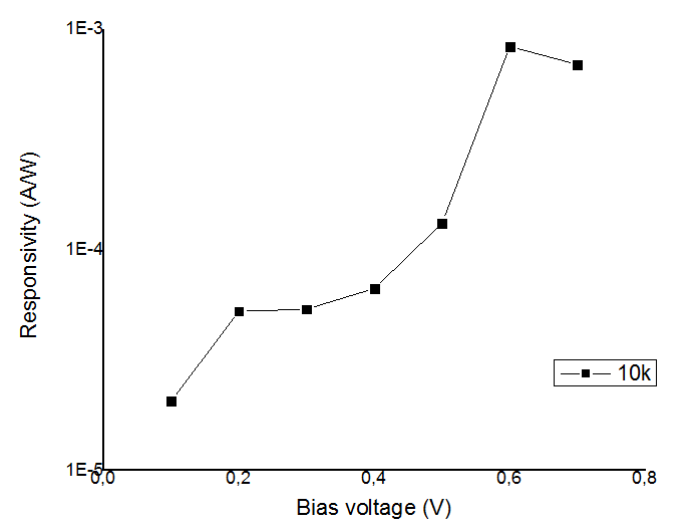

Figure 3-12: Black-body responsivity of sample \#3551 as a function of the bias voltage at $10 K$.

\section{3-2-5 Specific detectivity}

The detectivity is the signal over noise ratio and thus provides the final performance of the device, that is defined as:

$$
D=\frac{R}{i_{n}} \ldots \ldots \ldots \ldots(3-3)
$$

where $R$ and $i_{n}$ are respectively the responsivity and noise current of the device. In order to be able to compare the final performance of 2 detectors of different nature, it is interesting to remove from the data any dependence on their geometry and on the experimental parameters that were used in the measurements. As a consequence, the best way to compare 2 devices is to use the specific detectivity $\left(D^{*}\right)$ that is defined as [26]:

$$
D^{*}=D \sqrt{A \cdot \Delta f} \ldots \ldots \ldots \ldots(3-4)
$$

where $\mathrm{A}$ is the optical area of the device and $\Delta \mathrm{f}$ is the bandwidth of the noise measurements (divided by the number of sampling data of the FFT analyzer). 


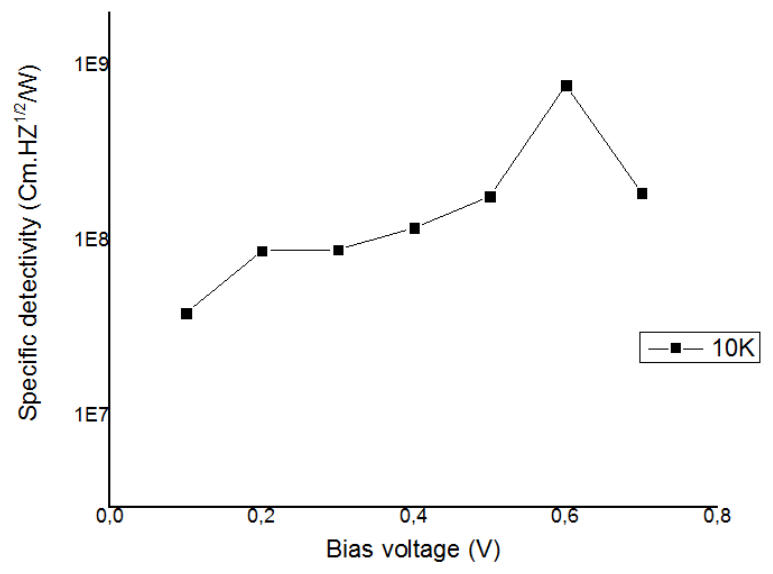

Figure 3-13 Specific detectivity of sample \# 3551.

We can see that, at $10 \mathrm{~K}$, the specific detectivity of sample SK \#3551 increases with bias up to a value of $7,69 \times 10^{8} \mathrm{~cm} \mathrm{~Hz}^{1 / 2} / \mathrm{W}$ (at bias = $0.6 \mathrm{~V}$ ), and then decreases, as expected, because of the rapid increase of the noise, observed in figure 3-9a [38].

Although it was not possible to calculate the detectivity of sample \#3601, a direct comparison with sample \#3551 would not be straightforward because they don't have exactly the same structure (the number of QD periods and the height of the AlGaAs barriers are different) [42] and no real conclusion about the efficiency of the SML-QDs with respect to the SK-QDs might be drawn. Still, we can suppose that the detectivity would be smaller due to the fact that the photocurrent was similar but the dark current (and noise) was larger in the SML QDIP investigated here. 


\section{Chapter 4: Photodetector based on submonolayer quantum dots in a quantum well.}

The results presented in the previous chapter showed that, although we expected SML-QDs to provide a better performance of the photodetectors (due to their stronger confinement and higher density), actually it seems to be worse in the case of a simple structure, probably because the activation energy of the SML-QDs is small and leads to a higher dark current and noise. As a consequence, we designed another structure with SML-QDs inserted in a GaAs/AlGaAs quantum well in order to increase the activation energy. The device (\# 3691) was processed and tested in the same manner as the previous ones and, as will be seen below, had a much better performance.

\section{4-1 Growth \& processing}

We have designed and grown a structure containing InAs/GaAs SML-QDs inserted in GaAs/AlGaAs quantum wells (QWs). The structure of the sample is shown in Figure 4-1. It consists of 2 thick Si-doped GaAs layers (contact layers) surrounding the active layer that contained 10 layers of SML-QDs, each of them inserted inside a $8.5 \mathrm{~nm}$ wide GaAs quantum well $\left(1.3 \mathrm{~nm}\right.$ of $\mathrm{GaAs}+6 \times(0.35+2.65) \times 2.83 \times 10^{-1} \mathrm{~nm}$ of SML-QDs $+2.1 \mathrm{~nm}$ of GaAs) having $45 \mathrm{~nm}$ thick $\mathrm{Al}_{0.1} \mathrm{Ga}_{0.9} \mathrm{As}$ barriers. Each SML-QD layer was built from the sequential deposition of $0.35 \mathrm{ML}$ of InAs followed by 2.65 MLs of GaAs, repeated 6 times in order to have SML-QDs with an approximate height and composition around $5.1 \mathrm{~nm}$ and $\mathrm{In}_{0.12} \mathrm{Ga}_{0.88} \mathrm{As}$, respectively. The contact layers and each thin GaAs layer covering the InAs SMLs were Si doped to $1 \times 10^{18}$ and $2 \times 10^{18} \mathrm{~cm}^{-3}$, respectively in order to have 2 electrons in the ground state of each QD, whose density was estimated to be around $4.5 \times 10^{11} \mathrm{~cm}^{-2}$ from the literature. Therefore, by doping each 2.65 ML-thick GaAs layer at $2 \times 10^{18} \mathrm{~cm}^{-3}$, the equivalent $2 \mathrm{D}$ electron density is thus $6 \times\left(2 \times 10^{18} \mathrm{~cm}^{-3} \times 2.65 \times 2.83 \times 10^{-8} \mathrm{~cm}\right)=$

$9.00 \times 10^{11} \mathrm{~cm}^{-2}$, which is 2 times the surface density of the islands (i.e. of the SML-QDs). 


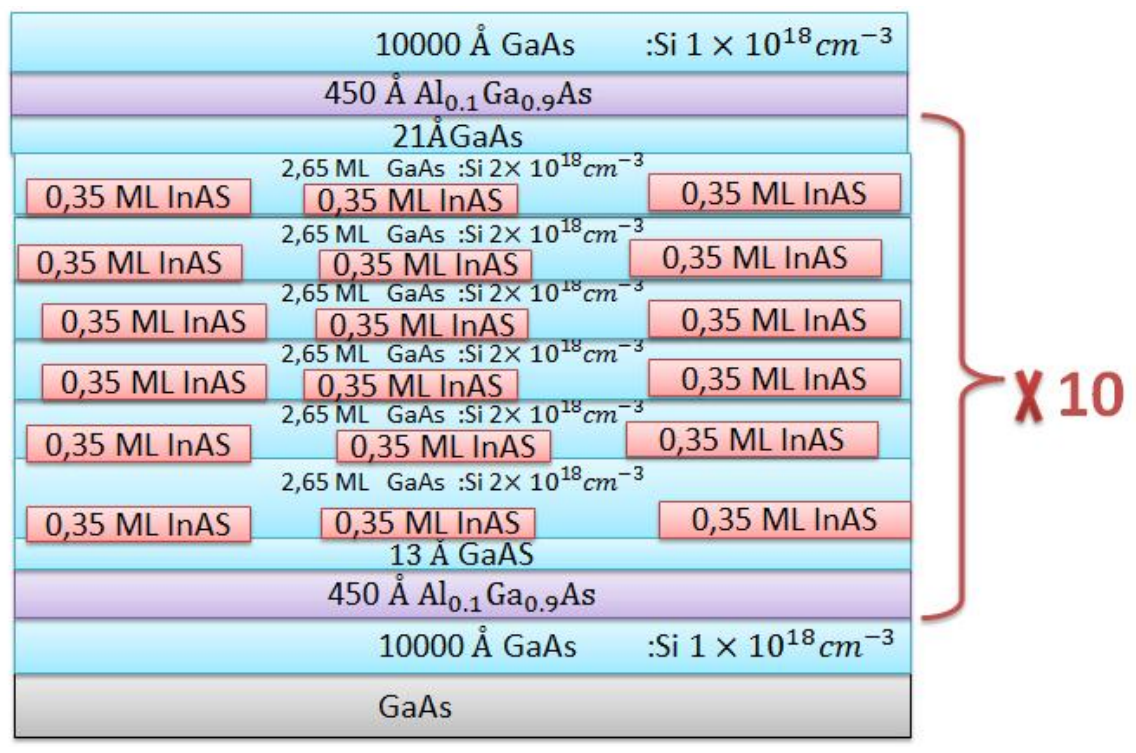

Figure 4-1: Schematics of the structure of sample \#3691.

As before, standard processing techniques were used to define small squared mesas using optical lithography, and metallization and RTA (Rapid Thermal Annealing) were used to make small Ohmic contacts of good quality. Finally, the sample was placed into a commercial chip carrier and the mesas were connected with thin gold wires (diameter $=$ $25 \mu \mathrm{m}$ ) to the pads of the chip carrier using a wire bonder (figure 4-2).

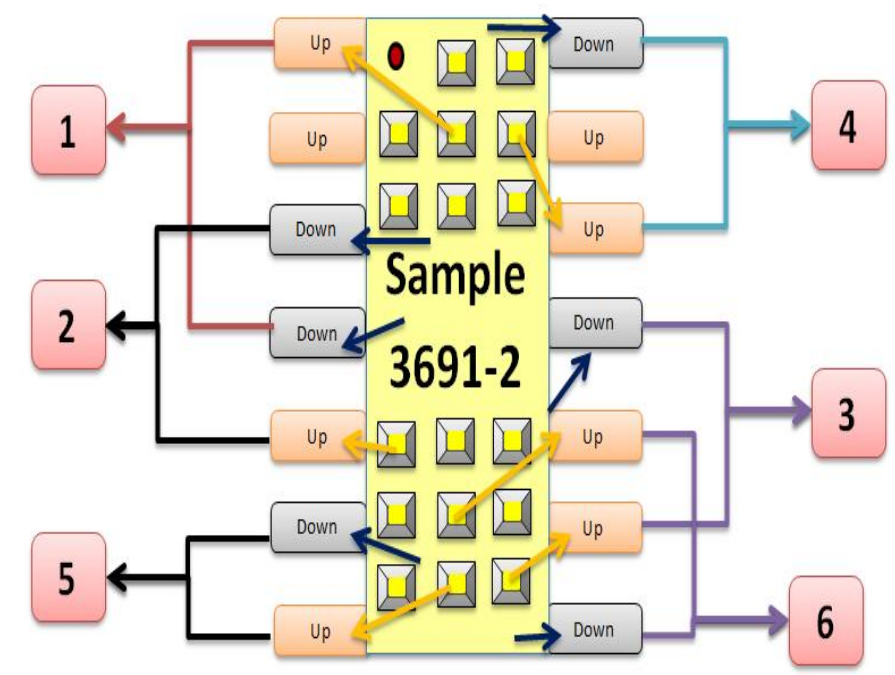

Figure 4-2: Schematics of the electrical connections between the devices and the pads. 


\section{4-2 Electro-optical characterization of the SML-QDWELL photodetector}

All the measurements presented in this chapter were performed again with the new $\mathrm{Cu}$ spring plate and showed some improvement with respect to the final temperature, when compared to the previous measurements.

\subsubsection{Dark Current}

In order to check the homogeneity of the processing and the reproducibility of the measurements, the dark current of 3 different mesas was measured, following the electrical connections illustrated in figure 4-2. From figure 4-3, which shows the results for 2 mesas, we can see that the data are very similar and confirm that the processing was performed in good conditions.

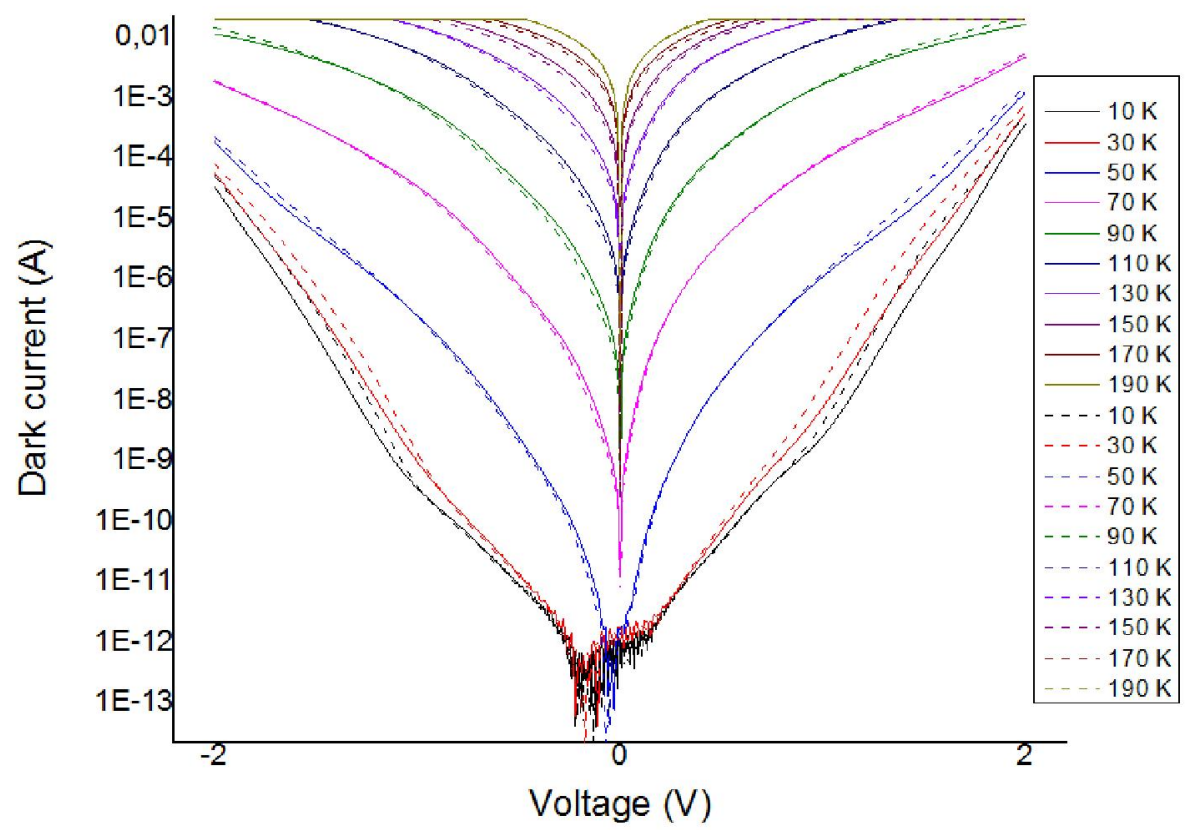

Figure 4-3: Dark current of two different mesas \#2 (full) and \#3 (dashed) of sample \#3691 at different temperatures.

In the rest of this chapter, mesa number \#2 was chosen to provide all the electro-optical data. Figure 4-4 shows that, below $30 \mathrm{~K}$, the dark current doesn't change significantly as a function of temperature, meaning that the main mechanism is most probably related to tunneling. At low bias, the plateau is due to the limitation of the experimental setup itself to measure lower current, while at higher bias, the exponential dependence of the 
current (which appears as a rather linear dependence in the logarithmic scale) is due to field-assisted tunneling through the top of the AlGaAs barriers that are distorted by the applied bias. As the temperature rises, the dark current increases considerably and is mostly due to thermal excitation, that dominates the spectrum, as expected above $50 \mathrm{~K}$.

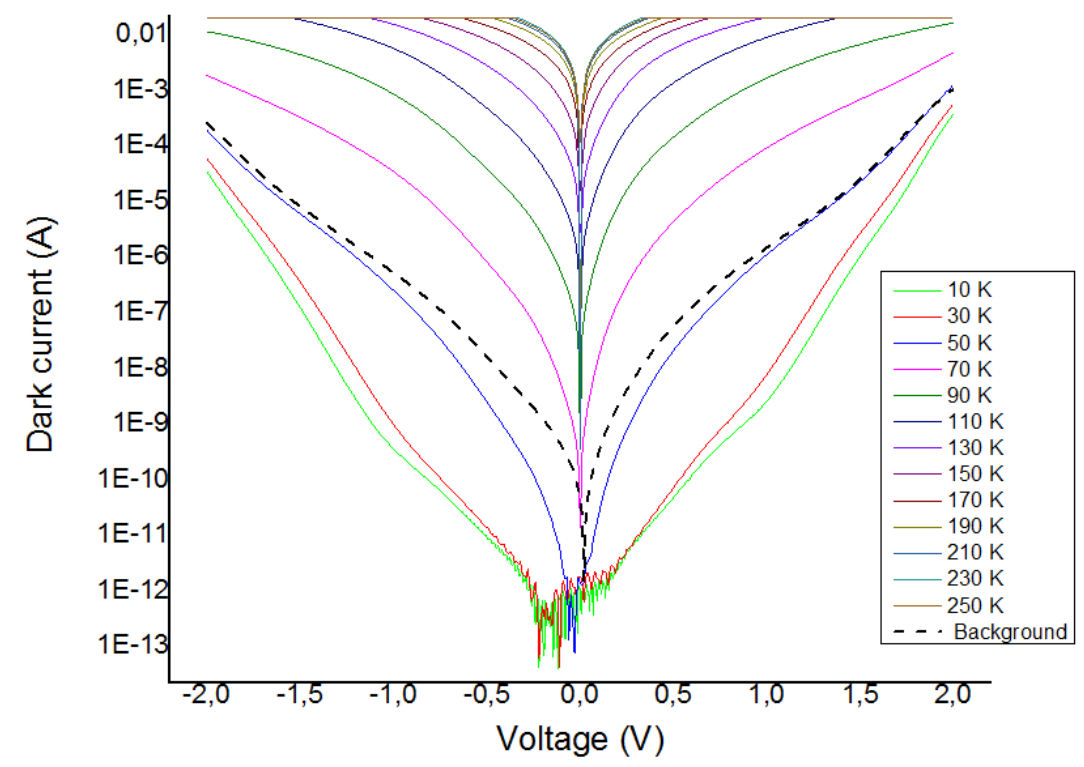

Figure 4-4: Dark current of mesa 2 of sample \#3691 showing also the I-V curve of the device obtained at $10 \mathrm{~K}$ when illuminated by a $300 \mathrm{~K}$ background (dashed, no $\mathrm{Cu}$ shield around the sample).

This rapid increase of the dark current can be easily seen by measuring the resistance of the device with a multimeter, which shows a resistance of $1.3 \mathrm{G} \Omega$ at $10 \mathrm{~K}$ and $30 \Omega$ at $250 \mathrm{~K}$. Figure $4-5$ shows the dark current as a function of temperature for a bias of $1 V$ in an Arrhenius plot. Since the thermal excitation of the carriers is ruled by an activation energy, the linear part of the figure can be fitted in order to estimate that activation energy from equation 3-2. 


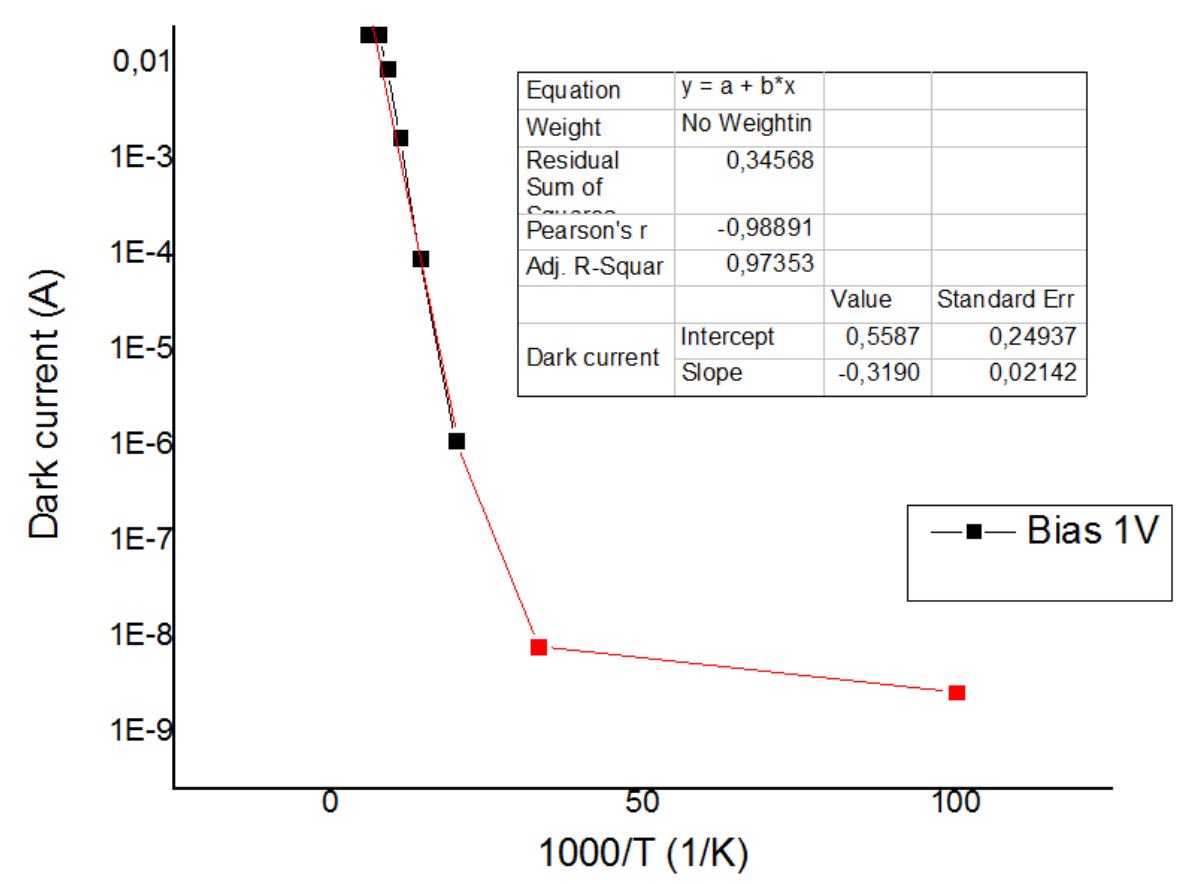

Figure 4-5: Dark current as a function of temperature at a bias of $1 \mathrm{~V}$.

The fitting procedure of the linear region of the graph provides a slope of $-0,319 \mathrm{~K}$. Therefore, the activation energy is $E_{\text {activ }}=63 \mathrm{meV}$ and corresponds to the difference of energy between the Fermi level of the structure and the top of the AlGaAs barriers. We can see that the activation energy is now higher than in sample \#3601, and is responsible for the lower dark current observed in the new device.

The dashed I-V curve in figure 4-4 was measured at $10 \mathrm{~K}$ without the $\mathrm{Cu}$ shield surrounding the sample (i.e. in the presence of a $300 \mathrm{~K}$ background scene) and shows that the device reaches BLIP conditions around $50-$ $60 \mathrm{~K}$ for all the bias values. It means that below that, temperature, the performance of the device is mainly limited by the flux of photons, while above that temperature it will be limited by the internal dark current.

\subsubsection{Noise current}

A photoconductor has several sources of noise: $1 / \mathrm{f}$ noise, Shot noise, Johnson noise (thermal noise), and generation-recombination (G-R) noise. In general, in a photodetector, the first 2 components are negligible, and the dominant noise is the G-R noise coming from the dark current and from the random absorption of photons by the detector [39]. The measurements 
are generally performed in the dark with the device covered by a shield (which acts also as a cold shield) to limit the absorption of background radiation. Figure 4-6 shows the noise current as a function of bias and temperature. We can notice that, in general, the noise curves show the same trends as the dark-current curves, which is consistent with the fact that the dark current is the main noise source. At 10 and $30 \mathrm{~K}$, the noise is very low at low bias voltage and shows a plateau which is due to the detection limit of the experimental setup around $3 \times 10^{-14} \mathrm{~A}$. For that temperature range, the noise is almost temperature independent and starts to increase at higher bias due to the increase of dark current by field-effect tunneling [39]. Above $30 \mathrm{~K}$, the thermal excitation of carriers becomes the dominant mechanism of dark current that increases rapidly as a function of temperature and leads to noise curves that behave in the same way.

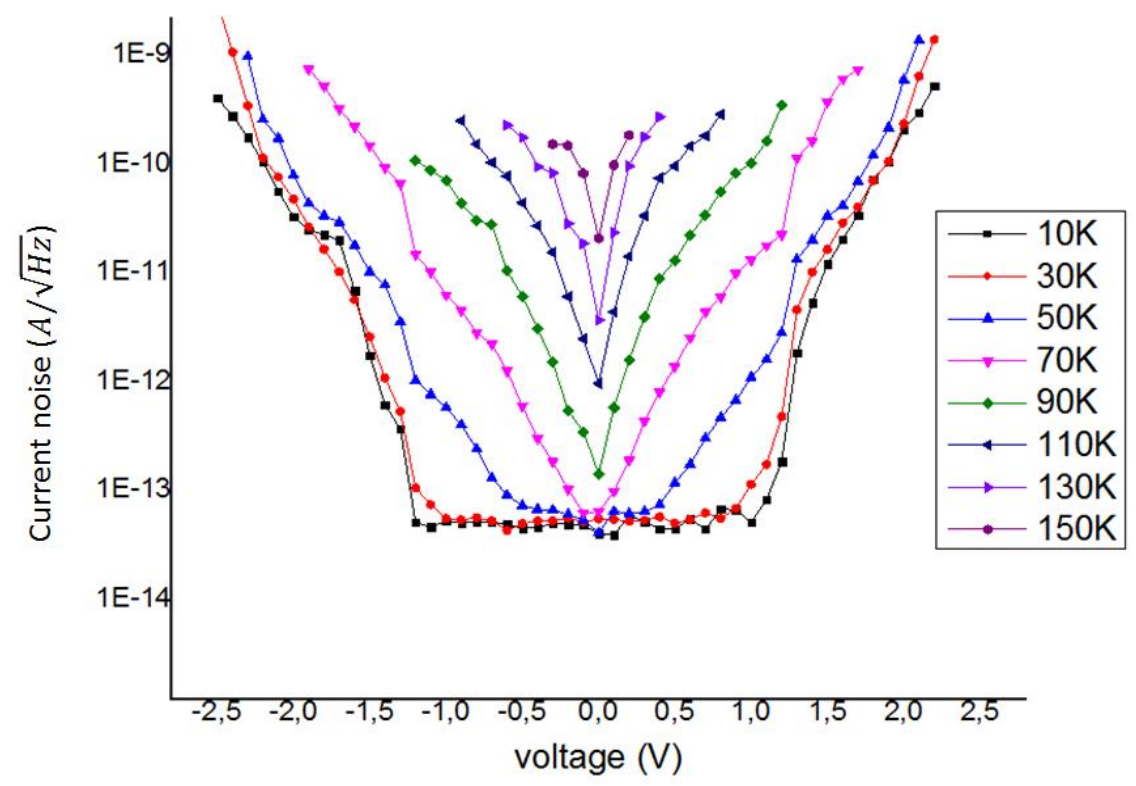

Figure 4-6: Noise of the current inside the detector as a function of bias voltage and temperature for sample \#3691. The intensity of the noise was normalized by the bandwidth of the measurements for the further calculation of the specific detectivity.

Figure 4-7 shows the noise current as a function of temperature at a bias of $1 V$. This bias was chosen because, as will be shown later, the performance of the device at $10 \mathrm{~K}$ was maximum for that bias value. 


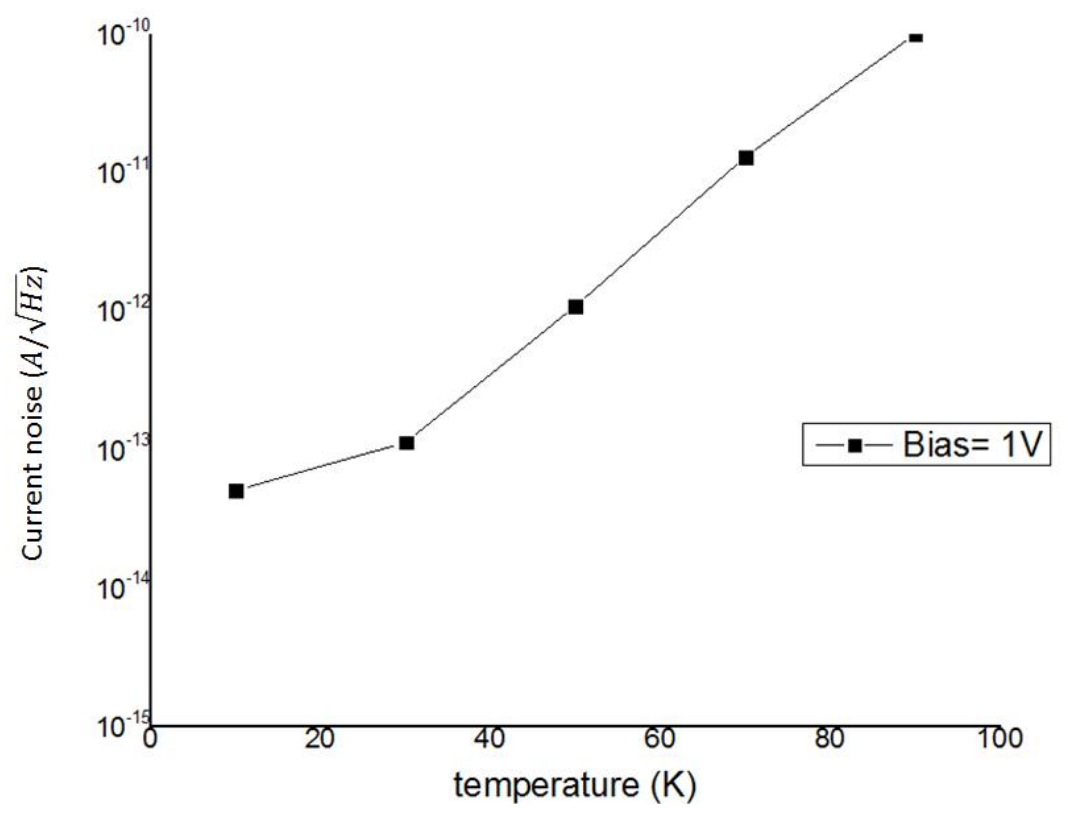

Figure 4-7: noise current as a function of temperature at bias $=1 \mathrm{~V}$

As can be seen from this figure, between 10 and $30 K$ the noise current was almost temperature independent, but above $30 \mathrm{~K}$, as the temperature rises, the noise increases considerably as a consequence of the strong increase of the dark current due to thermal excitation.

\subsubsection{Photocurrent with black body}

The photocurrent of the sample was measured again with a black body in the same conditions explained in section 3.2.3 and is shown in figure 4-8. As can be seen, all the curves are very similar over the whole bias and temperature range, confirming that the doping of the structure was adequate (2 electrons in each QD). Indeed, when the doping level of the nanostructures is not high enough, the photocurrent increases with rising temperature due to the filling of the ground-state energy level of the QDs (that is only partially populated) by thermally excited electrons. 


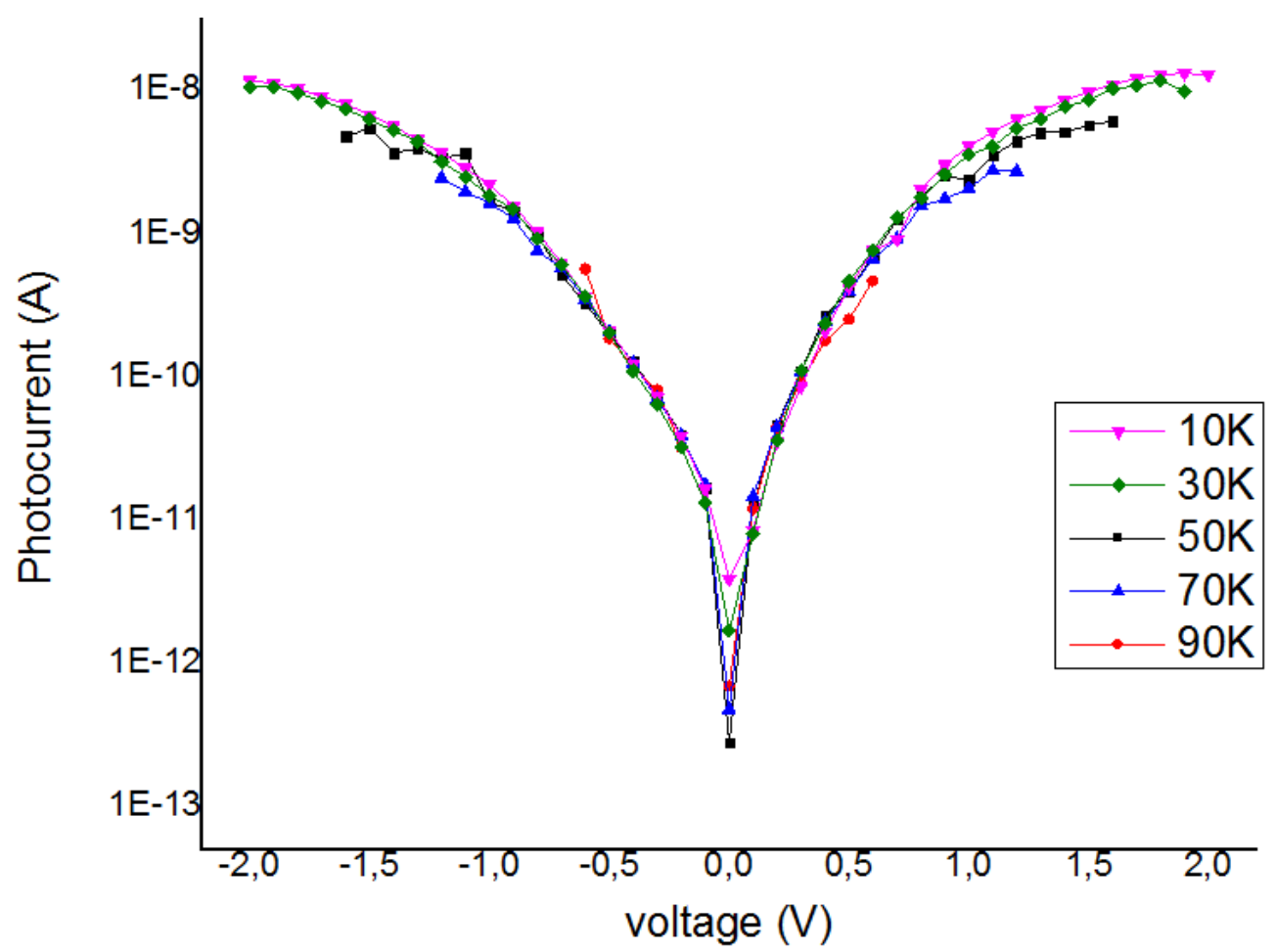

Figure 4-8: Photocurrent measurements as a function of bias and temperature using a black body.

\subsubsection{Spectral Response}

Fourier-transform infrared (FTIR) spectroscopy was used to investigate the optical spectral response of the photodetector. Figure 4-9 shows the normalized spectral response at $10 \mathrm{~K}$. We can see that the maximum signal for sample \#3691 at that temperature was for a bias of $1 \mathrm{~V}$. The peak at the opposite bias $(-1 \mathrm{~V})$ was lower, which means that the device is not exactly symmetric as can be seen in the original structure and as a consequence of some doping segregation and material intermixing that occur along the growth direction. 

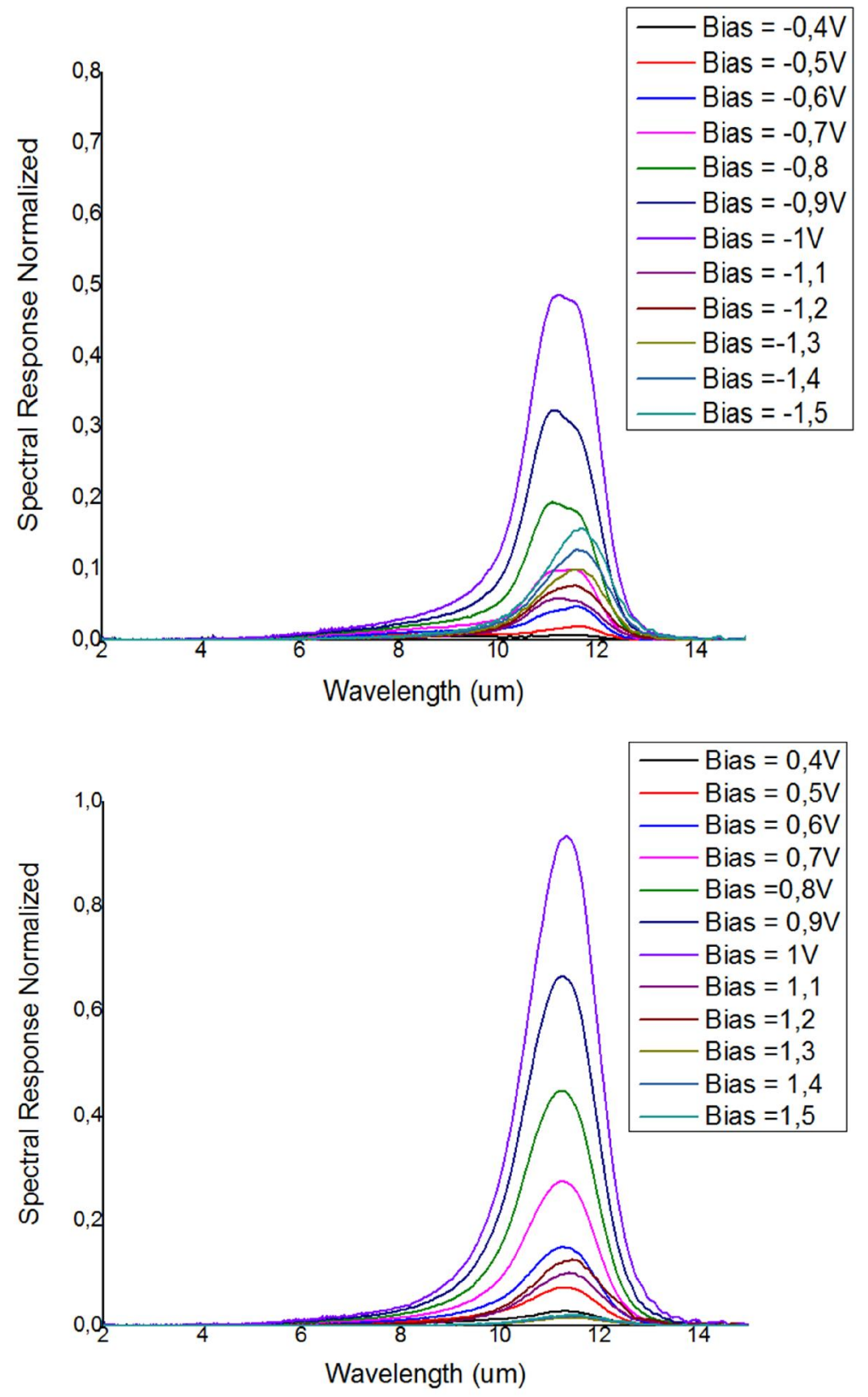

Figure 4-9: Spectral response (normalized) as a function of bias voltage at $10 \mathrm{~K}$ for positive and negative bias voltages.

The responsivity $R$ was calculated from all the available parameters and results, and then the specific detectivity $\left(D^{*}\right)$ was obtained. They are reported in figure 3-10 for sample \#3691 


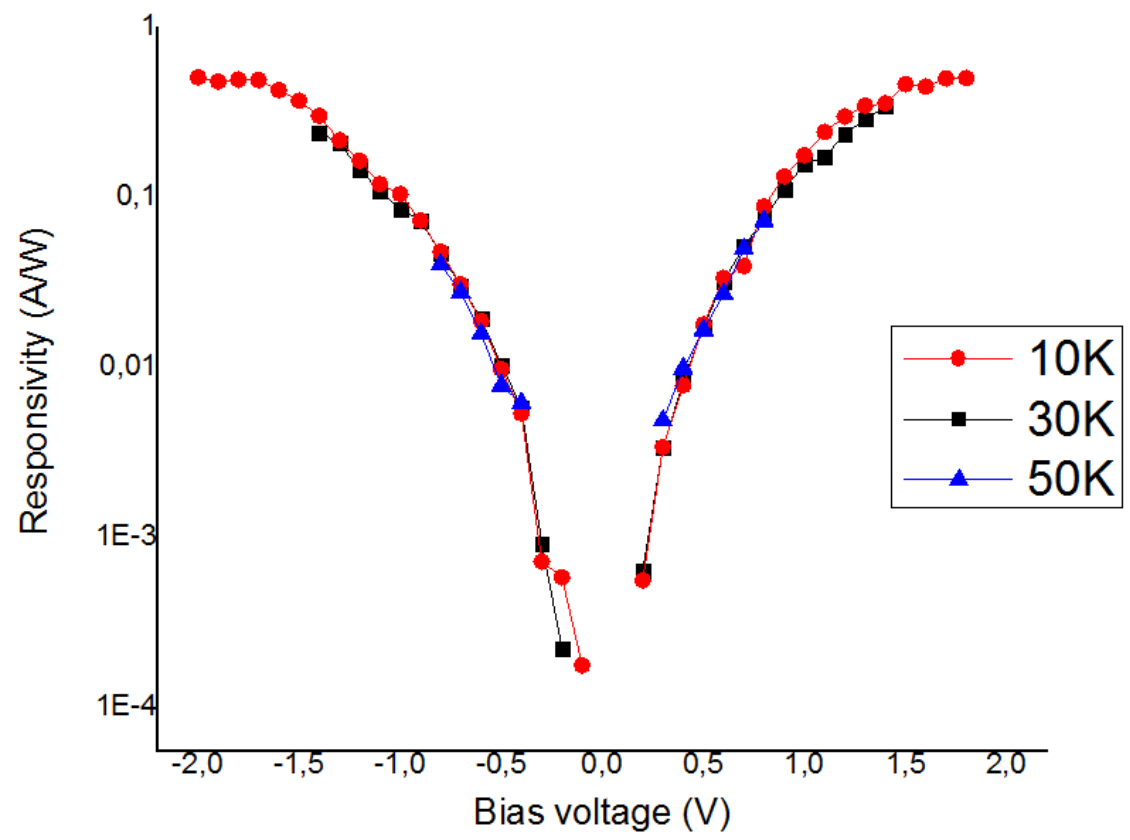

Figure 4-10: Black-body responsivity as a function of bias for different temperatures.

\section{4-2-5 Specific detectivity}

By using equation 3-4, the specific detectivity was obtained and is reported in figure 4-11.

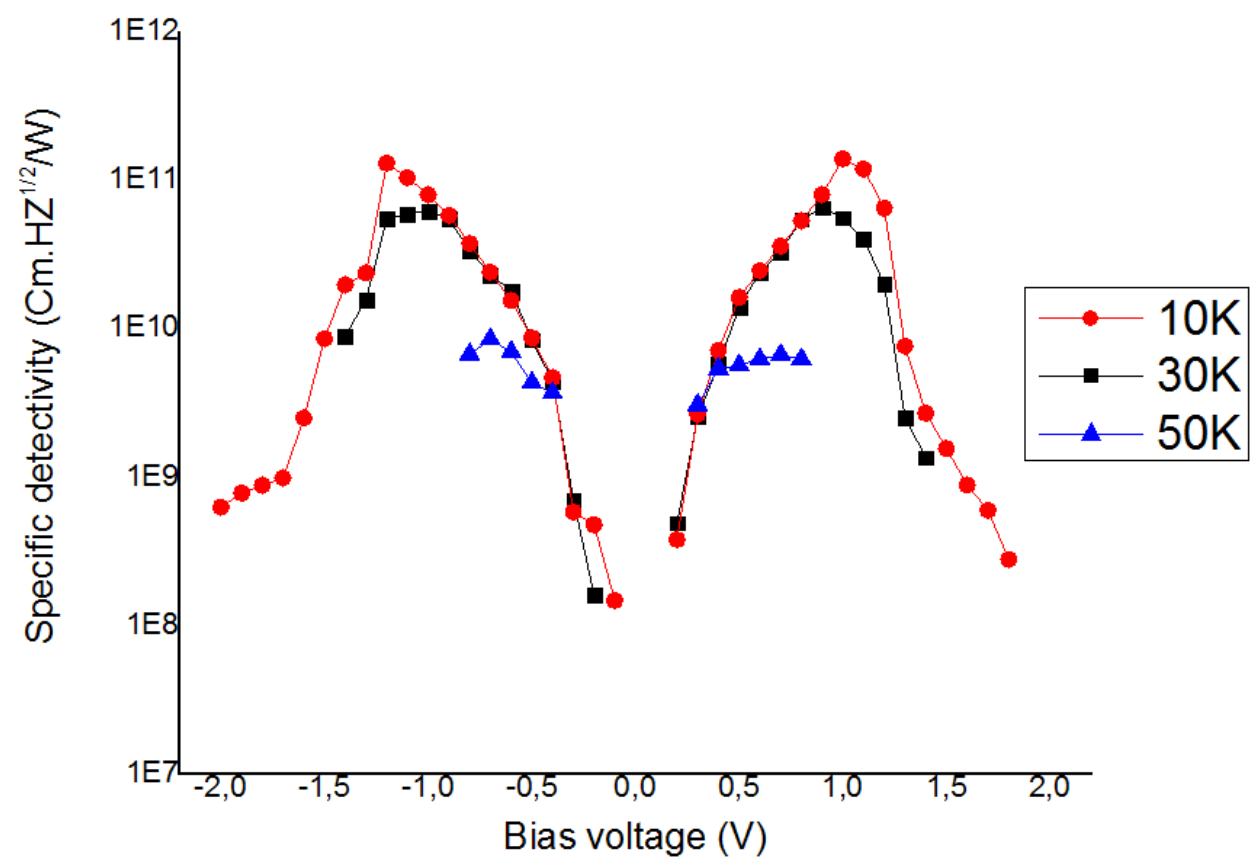

Figure 4-11: Specific detectivity as a function of bias for different temperatures. 
From figure 4-11 we can see that the highest specific detectivity value is $1,3810^{11} \mathrm{~cm} . \mathrm{Hz}^{1 / 2} / \mathrm{W}$ at a bias $=1 \mathrm{~V}$ and $\mathrm{T}=10 \mathrm{~K}$. Althgough the responsivity keeps increasing monotonically with the bias, the signal over noise ratio (i.e. the detectivity) has a maximum around $1 \mathrm{~V}$ because the noise is very small up to $1 \mathrm{~V}$ (floor of the setup) and then starts to increase abruptely with the bias. We also see that, although the responsivity is rather insensitive to temperature, the specific detectivity decreases quickly with rising temperatures [43] as a consequence of the strong increase of the noise current with temperature, as shown in figure 4-6. This value of the detectivity is the highest one ever obtained in our laboratory for any type of photodetector (containing quantum wells, Stranski-Krastanov quantum dots, submonolayer quantum dots or quantum cascade structures). To understand better the reasons why the SML-QDs inside a QW provided such good results, we compiled in Table 4 the advantages and drawbacks of the SK and SML quantum dots. In the first SML-QD sample (\#3601, with no QW), the overall performance was not good due to a very small activation energy $(42,8 \mathrm{meV})$ coming probably from the small size of the SML-QDs. In the second sample (\#3691), the SML-QDs were actually even smaller (stacks of 6 SMLs instead of 10 in the first sample) and therefore we could expect that the performance should even be worse due to the smaller difference between the ground state of the SML-QDs and the GaAs band edge. However, since those SML-QDs were inserted inside a GaAs/AlGaAs quantum well, the activation energy was actually calculated with respect to the top of the AlGaAs barrier of the QW which provides a higher activation energy. In addition, the first sample (\#3601) involved only bound-to-continuum transitions (from the ground state of the SMLQDs to the GaAs continuum), that have a lower oscillator strength than the bound-to-bound transitions involved in the second sample (\#3691) from the ground-sate of the SML-QDs to the excited state of the QW. The absorption of the radiation should thus be more efficient and, since the excited state of the QW was intentionally localized close to the top of the barrier, the escape of the photoexcited carriers was easy and provided a large photocurrent (responsivity). Another argument to explain why the second sample had a better performance comes from the literature: indeed, it seems that QDs containing stacks with more than 6 SMLs are probably worse due to some growth instabilities that allow larger QDs to collapse or merge with other ones [42]. 


\begin{tabular}{|l|l|}
\hline \multicolumn{1}{|c|}{ SK-QDs } & \multicolumn{1}{|c|}{ SML-QDs } \\
\hline $\begin{array}{l}\text { Have an InAs wetting layer, which } \\
\text { actually reduces the confinement of } \\
\text { the carriers and does not contribute } \\
\text { to the normal-incidence absorption. }\end{array}$ & $\begin{array}{l}\text { Have no wetting layer which leads to a } \\
\text { better quantum confinement and } \\
\text { reduces the strain. }\end{array}$ \\
\hline Density of QDs around $10^{10} \mathrm{~cm}^{-2}$ & Density of QDs up to $10^{12} \mathrm{~cm}^{-2}$ \\
\hline $\begin{array}{l}\text { Have one ground state and possibly } \\
\text { several excited states. }\end{array}$ & $\begin{array}{l}\text { Seem to have only one ground state } \\
\text { due to their smaller size. }\end{array}$ \\
\hline $\begin{array}{l}\text { Use around 2.2 ML of InAs per layer } \\
\text { of QDs. }\end{array}$ & $\begin{array}{l}\text { Can use a smaller amount of InAs per } \\
\text { layer of QD, which can help to stack } \\
\text { more layers. }\end{array}$ \\
\hline $\begin{array}{l}\text { Limited control of the height and } \\
\text { base of the QDs. }\end{array}$ & $\begin{array}{l}\text { Full control of the height of the QDs, } \\
\text { and some limited control on the } \\
\text { diameter. }\end{array}$ \\
\hline Very easy to grow. & $\begin{array}{l}\text { Need very specific (more difficult to } \\
\text { achieve) growth conditions. }\end{array}$ \\
\hline Table 4: Comparison of both types of QDs. & \\
\hline
\end{tabular}




\section{Conclusion}

Several types of infrared photodetectors based on InAs quantum dots (QDIPs) were compared. QDIPs based on conventional InAs quantum dots grown in the Stranski-Krastanov growth mode (SK-QDs) are well known and widely investigated, but their final performance suffer from several drawbacks like their small areal density $\left(1-5 \times 10^{10} \mathrm{~cm}^{-2}\right)$, the large strain energy due to the presence of the wetting layer, the weak lateral confinement resulting from the lens shape (and presence of the wetting layer), and the lack of size control due to their self-assembling nature. Submonolayer quantum dots (SML-QDs) are more difficult to grow, as they need very specific growth conditions to be formed, but they have a higher density (up to $10^{12} \mathrm{~cm}^{-2}$ ), have no wetting layer, have a smaller lateral size and a better 3D confinement, and can be grown with any height.

The devices investigated here contained both types of QDs and were processed in a clean room, using photolitography, wet etching, electronbeam metallization, rapid thermal annealing (RTA) and wire bonding. Then, they were tested using dark current (I-V) measurements, noise measurements with a spectrum analyzer, absorption measurements by FTIR, and responsivity measurements with a black body. All these measurements were performed at low temperature, and special care was taken to be sure that the samples were always in good thermal contact with the cold finger of the cryostat, using a special spring plate to press the chip carrier inside its socket and against the cold finger. The experimental results showed that, despite their high density, SML-QDs alone are not enough to improve the performance of the devices, since their small size and low average In content yield a blue shift of the energy levels which lowers the activation energy and increases considerably the dark current and noise with respect to usual SK-QDs. However, when the SML-QDs are inserted into a GaAs/AlGaAs quantum well (QW) having an excited state close to the top of the barrier, the absorption of the radiation is improved, due to the higher value of the oscillator strength of the transition from the level of the SML-QD to the excited state of the QW, and the escape of the carriers to the continuum is easy. In addition, the dark current is also reduced, leading thus to a very high specific detectivity which is 2 orders of magnitude larger than for the other devices. By 
optimizing the doping, density, size and composition of the SML-QDs, it should be possible to reach an even better performance. 


\section{Conferences}

A. Alzeidan, M.S. Claro and A.A. Quivy, "Infrared photodetectors based on submonolayer quantum dots", poster presented at the Physics Meeting 2016, Natal (RN, Brazil), September 3-7, 2016.

\section{Papers}

A. Alzeidan, M.S. Claro and A.A. Quivy, "High-detectivity infrared photodetector based on InAs submonolayer quantum dots", to be submitted to Applied Physics Letters. 


\section{Bibliography}

[1]: Introduction to Infrared Spectroscopy (IR), Royal Society of Chemistry (2009).

[2]: Alexander Weber, Intraband Spectroscopy of Semiconductor Quantum Dots, Physikalisches Institut der Julius-Maximilians-Universität Würzburg Institut Electronique Fondamentale, Orsay. August 1998

[3]: P. Martyniuk, A. Rogalski. Review Quantum-dot infrared photodetectors Status and outlook. Progress in Quantum Electronics 32 (2008) 89-120

[4]: J. Phillips, K. Kamath, T. Brock, and P. Bhattacharya. Characteristics of InAs/AlGaAs self-organized quantum dot modulation doped field effect transistors APPLIED PHYSICS LETTERS VOLUME 72, NUMBER 2629 JUNE 1998

[5]: Adrienne D. Stiff-Roberts, Quantum-dot infrared photodetectors: a review, Journal of nanophotonics. 3(1), 031607, (2009).

[6]: https://upload.wikimedia.org/wikipedia/commons/6/64/Infrared_spectrum.gif

[7]: Marcel Santos Claro: Determinação da corrente de escuro em fotodetectoresde radiação infravermelha baseados em poços quânticos (QWIPs), master Dissertation university of São Paulo-2012.

[8]: https://en.wikipedia.org/wiki/File:Black-body_realization.png

[9]: Stephen Gasiorowics, Quantum physics, University of Minnesota, John Wiley and Sons, Inc. new york. ISBN 0471057002

[10]: P.K. Mani, Black body radiation, planck's radiation, wien's law, stephen boltzmann law in remote sensing, PowerPoint, (2014).

[11]: VINCENT, J. D. Fundamentals of infrared detector operation and testing. New York: John Wiley \& Sons, 1990

[12]: http://www.eumetrain.org/data/3/30/navmenu.php?tab=5\&page=1.2.0

[13]: Michael Vollmer and Klaus-Peter Mollmann. Infrared Thermal Imaging. WILEY-VCH Verlag, 1st edition, 2010.

[14]: G.D. Boreman and E.L. Dereniak, "Infrared Detectors and Systems", ISBN 0471122092, WileyInterscience

[15]: A. Rogalski. Infrared Physics \& Technology 43 (2002) 187-210.

[16]: Antoni Rogalski, Infrared detectors: status and trends, Review, Progress in Quantum Electronics 27 (2003) 59-210.

[17]: Antoni Rogalski, 2011, Infrared Detectors, $2^{\text {nd }}$ edition, CRC Press, Taylor \& Francis Group, page 30.

[18]: M.A .Kinch, "Fundamental Physics of Infrared Detector Materials," Journal of Electronic Materials29, 809-17, 2000. 
[19]: Jie Zhang, Win-Ching Hung Quantum Well Infrared Detector, Department of Electrical and Computer Engineering. PowerPoint PPT Presentation.

[20]: Quantum wells, Quantum wires and Quantum dots Presented By_ To_ Sanjeeb Limbu(Reg.No:14305019) Dr.A.Subramania Sir M.Tech.-Nanoscience \& Technology Associate Professor (CNST).

[21]: Sarath D. Gunapala, Sumith V. Bandara, John K. Liu. Quantum Well Infrared Photodetector Technology and Applications.IEEEjournalVOL. 20, NO.6, (2014).

[22]: E.H.C.Parker, the technology and physics of molecular beam epitaxy, ISBN: 0-30641860-6, Plenum Press, New York, 1985.

[23]: https://en.wikipedia.org/wiki/File:MBE.svg.

[24]: M. Sugarawa, Self-assembled InGaAs/GaAs quantum dots, Volume 60, Academic Press,1999.ISBN: 9780080864587.

[25]: F. Hopfer, A. Mutig, M. Kuntz, G. Fiol, D. Bimberg, N. N. Ledentsov, V. A. Shchukin, S. S. Mikhrin, D. L. Livshits, I. L. Krestnikov, A. R. Kovsh, N. D. Zakharov, and P. Werner, Single-mode submonolayer quantum-dot vertical-cavity surface-emitting lasers with high modulation bandwidth, Appl. Phys. Lett. 89, 141106 (2006).

[26]: S. Sengupta, J. O. Kim, A. V. Barve, S. Adhikary, Y. D. Sharma, N. Gautam, S. J. Lee, S. K. Noh, S. Chakrabarti, and S. Krishna. Sub-monolayer quantum dots in confinement enhanced dots-in-a-well heterostructure. Appl. Phys. Lett. 100, 191111 (2012).

[27]: Álvaro Diego B. Maia, Crescimento, fabricação e teste defotodetectores de radiaçãoinfravermelha baseados em pontosquânticos. PhD Dissertation university of São Paulo-2012.

[28]: P. Martyniuk, A. Rogalski, Quantum-dot infrared photodetectors:

Status and outlook, Review.Progress in Quantum Electronics 32 (2008) 89-120.

[29]: Kinch, M. A. Fundamentals of infrared detector materials, ISBN 978-0-8194-6731-7, Bellingham, Washington USA, 2007.

[30]: Laboratório de Novos Materiais Semicondutores Grupo de Epitaxia por Feixe Molecular (MBE) IFUSP. http://portal.if.usp.br/mbe/pt-br

[31]: H. Schneider, H. C. Liu, Quantum-well infrared photodetectors, ISBN -103-540-

36323-8 Springer, Berlin, (2007).

[32]: Keithley, Manual do equipamentoKeithley 428 Current Amplifier.

[33]: Jiaui Shao, Engineered quantum dots for infrared photodetectors. PhD dissertation, University of New Mexico. December2011

[34]: Arvind Pawan Ravi kumar, II-VI Materials-Based High Performance Intersubband Devices. PhD dissertation, September 2015. 
[35]: Noise Analysis in Operational Amplifier Circuits, Application Report, Digital Signal Processing Solutions (2007).

[36]: Z. M. Ye, J. C. Campbell, Z. H. Chen, E. T. Kim, and A. Madhukar, J. Appl. Phys.92, 7462 (2002).

[37]: Eui-Tae Kim, Anupam Madhukar, Zhengmao Ye, and Joe C. Campbell, High detectivity InAs quantum dot infrared photodetectors, J. Appl. Phys.84, 3277 (2004).

[38]: Z. M. Ye, J. C. Campbell, Z. H. Chen, E. T. Kim, and A. Madhukar, IEEE J. Quantum Electron.38, 1234 (2002).

[39]: Zhengmao Ye, Joe C. Campbell, Zhonghui Chen, Eui-Tae Kim, and Anupam Madhukar. Noise and photoconductive gain in InAs quantum-dot. J. Appl. Phys.83, 1234 (2003).

[40]: Maia, A. D. B, da Silva, E. C. FQuivy, A. A, Bindilatti, V, de Aquino, V. M, Dias, I. F. L, Simulation of the electronic properties of InxGa1-xAs quantum dots and their wetting layer under the influence of indium segregation, J. Appl. Phys.114, 083708 (2013).

[41]: Maia, A. D. B, da Silva, E. C. F, Quivy, A. A, et al. The influence of different indiumcomposition profiles on the electronic structure of lens-shaped InxGa1-xAs quantum dots, J. Appl. Phys.45, 225104 (2012).

[42]: J. O. Kim, S. Sengupta, A. V. Barve, Y. D. Sharma, S. Adhikary, S. J. Lee, S. K. Noh, M. S. Allen, J. W. Allen, S. Chakrabarti, and S. Krishna. Multi-stack InAs/InGaAs submonolayer quantum dots infrared photodetectors, J. Appl. Phys.102, 011131 (2013).

[43]: A. D. Stiff, S. Krishna, P. Bhattacharya, and S. Kennerly. High-detectivity, normalincidence, mid-infrared,,InAs/GaAs quantum-dot detector operating at $150 \mathrm{~K}$. J. Appl. Phys.79, 421 (2001).

[44]: S. Dhomkar, N. Vaxelaire, H. Ji, V. Shuvayev, M. C. Tamargo, I. L. Kuskovsky, and I. C. Noyan, Determination of shape anisotropy in embedded low contrast submonolayer quantum dot structures, Appl. Phys. Lett. 107, 251905 (2015).

[45]: Sandro Martini, Estudo da segregacao de Indio em camadas Epitaxiais de ÍnGuAS crescidas sobre substratos de Ga As (001). PhD Dissertation university of São Paulo 2002.

[46]: Marcel Santos Claro, novas tecnologias para deteccao infravermelha de alto desempenho, PhD Dissertation university of São Paulo - 2017. 